\title{
On the Initial-value Problems with Data on a Characteristic Surface for Linear Systems of First Order Equations
}

\author{
By
}

Masatake Miyake*

\section{§. Introduction and Definitions}

We study in this paper the following initial-value problem

$$
\begin{gathered}
A(t, x) \frac{\partial}{\partial t} \vec{u}(t, x)+\mathscr{L}\left(t, x ; \frac{\partial}{\partial x}\right) \vec{u}(t, x)=\vec{f}(t, x), \\
\left.\vec{u}\right|_{t=0}=\vec{U}(x), \quad t \in R^{1}, x=\left(x_{1}, \ldots, x_{n}\right) \in R^{n},
\end{gathered}
$$

where $\mathscr{L}\left(t, x ; \frac{\partial}{\partial x}\right)=\sum_{k=1}^{n} B_{k}(t, x) \frac{\partial}{\partial x_{k}}+C(t, x) . \quad A, B_{k}$ and $C$ are $N \times N$ matrices, $\vec{u}$ and $\vec{f}$ are vectors with $N$-components, i.e., $\vec{u}={ }^{t}\left(u_{1}, \ldots, u_{N}\right)$ and $\vec{f}={ }^{t}\left(f_{1}, \cdots, f_{N}\right)$.

Throughout this paper we study the problem in the class of analytic functions. More precisely we assume that the coefficients, initial-data (1.2), $\vec{f}$ and solutions are analytic functions in a neighborhood of the origin.

We assume that the initial surface $t=0$ is characteristic of the system (1.1), namely

$$
\operatorname{det} A(0, x)=0
$$

Definition 1.1. We say that the initial surface $t=0$ is simple char-

Received May 10, 1972.

Communicated by S. Matsuura.

* Department of Mathematics, Kanazawa University, Kanazawa, Japan. 
acteristic if for a positive integer $r$,

$$
\operatorname{rank} A(0, x)=N-r, \sum_{|\alpha|=r}\left|\left(\frac{\partial}{\partial \xi}\right)^{\alpha} H(0, x ; 1,0)\right| \neq 0
$$

where $H(t, x ; \tau, \xi)=\operatorname{det}\left(A(t, x) \tau+\sum_{k=1}^{n} B_{k}(t, x) \xi_{k}\right)$.

Now we remark that (1.3) is equivalent to that $h_{r}(0, x ; \xi)$ is not zero as a homogeneous polynomial in $\xi$ of degree $r$, where $H(0, x ; \tau, \xi)=$ $h_{r}(0, x ; \xi) \tau^{N-r}+h_{r+1}(0, x ; \xi) \tau^{N-r-1}+\cdots+h_{N}(0, x ; \xi)$.

Definition 1.2. We say that the initial surface $t=0$ is double characteristic if for a positive integer $r$,

$$
\begin{aligned}
& \operatorname{rank} A(0, x)=N-r, \sum_{|\alpha|=r}\left|\left(\frac{\partial}{\partial \xi}\right)^{\alpha} H(0, x ; 1,0)\right|=0, \\
& \sum_{|\alpha|=r+1}\left|\left(\frac{\partial}{\partial \xi}\right)^{\alpha} H(0, x ; 1,0)\right| \neq 0 .
\end{aligned}
$$

This condition is equivalent to that $h_{r}(0, x ; \xi)$ is zero as a polynomial in $\xi$ and $h_{r+1}(0, x ; \xi)$ is not zero as a polynomial in $\xi$.

In section 2 , we study the initial-value problem in the case of simple characteristic. From section 3 on, we study the problem in the case of double characteristic. In section 4 we state the main theorems under the assumption (3. Ass.) when $r \geqq 2$. In appendix we study the case with a certain assumption other than (3. Ass.). In section 8 , we give only the results in the case where $r=1$.

The arguments in this paper may be used for the initial-value problems of higher order system.

Our method relies essentially on the paper of Y. Hasegawa [6].

I. G. Petrowsky [12] and S. Mizohata [8] had proved the existence of null solutions and S. Matsuura [11] had proved the existence of null solutions for overdetermined system with constant coefficients (see also L. Hörmander [7]).

Similar problems were investigated by J. Vaillant [9], [10] and Y. Hasegawa [6]. Especially Mrs. Hasegawa treated the initial-value problems for the single equations. In the case where $r=1$, our results are 
similar to those of $[6]$.

Finally, the author wishes to thank Professor Mizohata and Mrs. Hasegawa for their valuable suggestions and constant encouragements.

\section{§2. Simple Characteristic Initial-value Problem}

In this section we review the reasoning given in the paper of G.F.D. Duff [1]. Without loss of generality we may assume

$$
\operatorname{rank} A(0, x)=N-r, \frac{\partial^{r} H}{\partial \xi_{1}^{r}}(0, x ; 1,0) \neq 0,(r \geqq 1) .
$$

Let $V(x)\left(C C^{N}\right)$ be the image of ${ }^{t} A(0, x)$ and $K(x)$ be the kernel of ${ }^{t} A(0, x)$, then we have $\operatorname{dim} V(x)=N-r$ and $\operatorname{dim} K(x)=r$. Let $\left\{\vec{b}_{i}(x)\right\}_{i=r+1}^{N}$ be a base of $V(x)$ and $\vec{a}_{i}(x),(i=r+1, \ldots, N)$ be an inverse image of $\vec{b}_{i}(x)$, i. e.,

$$
{ }^{t} A(0, x) \vec{a}_{i}(x)=\vec{b}_{i}(x), \quad(i=r+1, \cdots, N) .
$$

Let $\left\{\vec{l}_{i}(x)\right\}_{i=1}^{r}$ be a base of $K(x),{ }^{1)}$ then we have

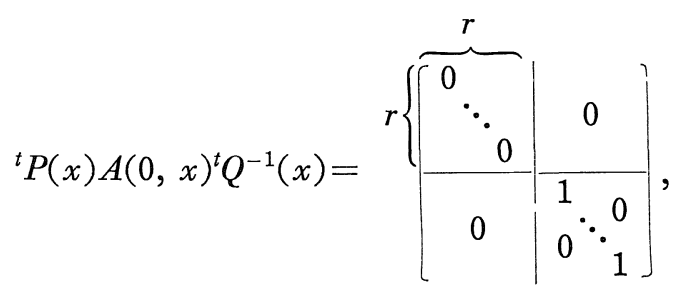

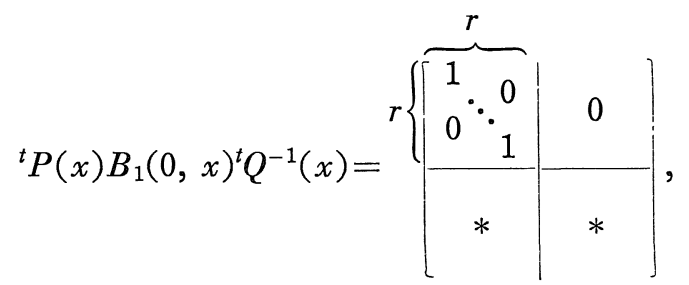

where $P(x)=\left(\vec{l}_{1}(x), \ldots, \vec{l}_{r}(x), \vec{a}_{r+1}(x), \ldots, \vec{a}_{N}(x)\right)^{2)}$ and $Q(x)=\left(\vec{b}_{1}(x), \ldots\right.$,

1) $\vec{a}_{i}(x), \vec{b}_{\imath}(x)$ and $\vec{l}_{\imath}(x)$ are determined analytically at $x$ in a neighborhood of the origin.

2) $\left(\vec{c}_{1}(x), \ldots, \vec{c}_{N}(x)\right)$ denotes the matrix with $i$-th column vector $\vec{c}_{\imath}(x)$. 
$\left.\vec{b}_{N}(x)\right)$, and $\vec{b}_{i}(x)={ }^{t} B_{1}(0, x) \vec{l}_{i}(x),(i=1, \ldots, r)$, since the assumption $(1.3)^{\prime}$ implies $\operatorname{dim}{ }^{t} B_{1}(0, x)(K(x)) / V(x)=r$.

Now we transform the unknown function $\vec{u}(t, x)$ to $\vec{v}(t, x)$ in a neighborhood of the origin by

$$
\vec{v}(t, x)={ }^{t} Q(x) \vec{u}(t, x) .
$$

We rewrite the system (1.1) in the following form

$$
\begin{gathered}
A(0, x) \frac{\partial}{\partial t} \vec{u}+\sum_{k=1}^{n} B_{k}(0, x) \frac{\partial}{\partial x_{k}} \vec{u}+t\left\{\tilde{A}(t, x) \frac{\partial}{\partial t}\right. \\
\left.+\sum_{k=1}^{n} \tilde{B}_{k}(t, x) \frac{\partial}{\partial x_{k}}\right\} \vec{u}+C(t, x) \vec{u}=\vec{f} .
\end{gathered}
$$

Then we have the following system equivalent to (1.1);

$$
\begin{aligned}
& \left.\frac{\partial}{\partial x_{1}} v_{i}=\sum_{j=1}^{N} b_{i j}\left(t, x ; t \frac{\partial}{\partial t}, t \frac{\partial}{\partial x_{1}}, \frac{\partial}{\partial x^{\prime}}\right) v_{j}+<\vec{l}_{i}, \vec{f}\right\rangle, \quad(i=1, \ldots, r) \\
& \frac{\partial}{\partial t} v_{i}=\sum_{j=1}^{N} b_{i j}\left(t, x ; t \frac{\partial}{\partial t}, \frac{\partial}{\partial x}\right) v_{j}+<\vec{a}_{i}, \vec{f}>, \quad(i=r+1, \ldots, N)
\end{aligned}
$$

where $x^{\prime}=\left(x_{2}, \cdots, x_{n}\right) \in R^{n-1}$ and $b_{i j}\left(t, x ; t \tau, t \xi_{1}, \xi^{\prime}\right),(i=1, \ldots, r)$ are polynomials in $\tau, \xi_{1}$ and $\xi^{\prime}=\left(\xi_{2}, \ldots, \xi_{n}\right)$ of degree $1 .<,>$ denotes the scalar product.

Theorem 2.1. Under the assumption (1.3), the following conditions are necessary and sufficient for the existence of a solution of the problem (1.1)-(1.2) in a neighborhood of the origin:

$$
<\vec{l}_{i}(x), \mathscr{L}\left(0, x ; \frac{\partial}{\partial x}\right) \vec{U}(x)-\vec{f}(x)>=0, \quad(i=1, \ldots, r) .
$$

For the proof of Theorem 2.1 we use the following lemma.

Lemma 2.1. (Goursat-problem) Let us consider the following problem to the system (2.4), then there exists a unique solution in a neighborhood of the origin:

$$
\left.v_{i}\right|_{x_{1}=0}=V_{i}\left(t, x^{\prime}\right), \quad(i=1, \ldots, r)
$$




$$
\left.v_{i}\right|_{t=0}=V_{i}(x), \quad(i=r+1, \ldots, N) .
$$

For the proof of the lemma, see $[1],[2]$ and $[9]$. Or, we can prove it in the same way as Proposition 5.1.

Proof of Theorem 2.1. Since the system (1.1) is equivalent to (2.4), we prove the theorem for (2.4). Let $\vec{V}(x)={ }^{t}\left(V_{1}(x), \ldots, V_{N}(x)\right)$ be the initial-data of (2.4), i.e., $\vec{V}(x)={ }^{t} Q(x) \vec{U}(x)$, then the condition (2.5) is transformed into

$$
\begin{gathered}
\frac{\partial}{\partial x_{1}} V_{i}(x)=\sum_{j=1}^{N} b_{i j}\left(0, x ; 0,0, \frac{\partial}{\partial x^{\prime}}\right) V_{j}(x)+<\vec{l}_{i}(x), \vec{f}(0, x)>, \\
(i=1, \ldots, r) .
\end{gathered}
$$

Since the necessity is clear, we prove the sufficiency. Now let us consider the following Goursat-problem to the system (2.4);

$$
\begin{array}{ll}
\left.v_{i}\right|_{x_{1}=0}=V_{i}\left(0, x^{\prime}\right)+t \chi_{i}\left(t, x^{\prime}\right), & (i=1, \ldots, r) \\
\left.v_{i}\right|_{t=0}=V_{i}(x), & (i=r+1, \ldots, N),
\end{array}
$$

where $x_{i}\left(t, x^{\prime}\right)$ are arbitrary analytic functions in $t$ and $x^{\prime}$. Then Lemma 2.1 asserts the existence of a unique solution $v_{i}(t, x),(i=1, \ldots, N)$ in a neighborhood of the origin. Let us show that this solution satisfies $v_{i}(0, x)=V_{i}(x),(i=1, \cdots, r)$ in a neighborhood of the origin. At first we note that $v_{i}(0, x),(i=1, \cdots, r)$ should be a solution of the following initialvalue problem;

$$
\begin{gathered}
\frac{\partial}{\partial x_{1}} v_{i}(0, x)=\sum_{j=1}^{N} b_{i j}\left(0, x ; 0,0, \frac{\partial}{\partial x^{\prime}}\right) v_{j}(0, x)+<\vec{l}_{i}(x), \vec{f}(0, x)> \\
\left.v_{i}(0, x)\right|_{x_{1}=0}=V_{i}\left(0, x^{\prime}\right), \quad(i=1, \cdots, r),
\end{gathered}
$$

where $v_{i}(0, x)=V_{i}(x),(i=r+1, \cdots, N)$.

On the other hand, $V_{i}(x),(i=1, \ldots, r)$ is also a solution of (2.8) in view of (2.6). Then we have $v_{i}(0, x)=V_{i}(x),(i=1, \ldots, r)$ in a neighborhood of the origin.

Finally the non-uniqueness of the solution is obvious from the arbitrariness of $\chi_{i}\left(t, x^{\prime}\right)$ in $(2.7)$.

Q.E.D. 


\section{§3. Reduction to the Standard Form}

To study the double characteristic case we reduce the system (1.1) to the standard form as in $\S 2$. Without loss of generality we may assume

$$
\operatorname{rank} A(0, x)=N-r, \sum_{|\alpha|=r}\left|\left(\frac{\partial}{\partial \xi}\right)^{\alpha} H(0, x ; 1,0)\right|=0
$$

$$
\frac{\partial^{r+1} H}{\partial \xi_{1}^{r+1}}(0, x ; 1,0) \neq 0 .
$$

The assumption (1.4)' implies immediately

$$
\begin{aligned}
& \operatorname{dim}{ }^{t} B_{k}(0, x)(K(x)) / V(x) \leqq r-1, \quad(k=2, \cdots, n) \\
& \operatorname{dim}{ }^{t} B_{1}(0, x)(K(x)) / V(x)=r-1 \\
& { }^{t} B_{1}(0, x) \vec{l}_{12}(x) \neq 0
\end{aligned}
$$

where $\vec{l}_{12}(x)(\in K(x))$ is a null vector of ${ }^{t} B_{1}(0, x)$ modulo $V(x)$, i.e., ${ }^{t} B_{1}(0, x) \vec{l}_{12}(x) \in V(x)$.

We shall prove only that ${ }^{t} B_{1}(0, x) \vec{l}_{12}(x) \neq 0$, since the others are proved by the same reasoning. It suffices to prove that ${ }^{t} B_{1}(0,0) \vec{l}_{12}(0) \neq$ 0 . We now assume that ${ }^{t} B_{1}(0,0) \vec{l}_{12}(0)=0$. If we put $P_{3}=\left(\vec{l}_{12}(0), \vec{l}_{23}\right.$, $\left.\ldots, \vec{l}_{r 3}, \vec{a}_{r+1}(0), \ldots, \vec{a}_{N}(0)\right)$ and $Q_{3}=\left(\vec{b}_{13}, \ldots, \vec{b}_{r 3}, \vec{b}_{r+1}(0), \ldots, \vec{b}_{N}(0)\right)$, where we choose $\left\{\vec{l}_{i 3}\right\}_{i=2}^{r}(C K(0))$ and $\left\{\vec{b}_{i 3}\right\}_{i=1}^{r}$ so that $\left\{\vec{l}_{12}(0), \vec{l}_{23}, \ldots, \vec{l}_{r 3}\right\}$ is a base of $K(0)$ and $\left\{\vec{b}_{13}, \ldots, \vec{b}_{r 3}, \vec{b}_{r+1}(0), \ldots, \vec{b}_{N}(0)\right\}$, is a base of $C^{N}$. Then we have

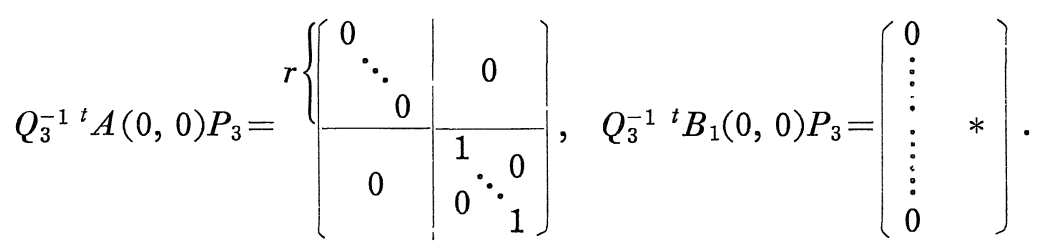

We now define a modified characteristic polynomial $L_{1}(\tau, \xi)$ by

$$
L_{1}(\tau, \xi) \equiv \operatorname{det}\left(Q_{3}^{-1}\left({ }^{t} A(0,0) \tau+\sum_{k=1}^{n}{ }^{t} B_{k}(0,0) \xi_{k}\right) P_{3}\right)
$$




$$
=\frac{\operatorname{det} P_{3}}{\operatorname{det} Q_{3}} H(0,0 ; \tau, \xi) .
$$

Then we have that the coefficient of $\xi_{1}^{r+1} \tau^{N-r-1}$ of $L_{1}(\tau, \xi)$ is zero. This contradicts $(1.4)^{\prime}$.

Then there exists a vector $\vec{a}_{r+1,2}(x) \in\left\{\vec{a}_{r+1}(x), \ldots, \vec{a}_{N}(x)\right\}$ such that

$$
{ }^{t} A(0, x) \vec{a}_{r+1,2}(x)={ }^{t} B_{1}(0, x) \vec{l}_{12}(x),
$$

where $\left\{\vec{a}_{r+1}, \ldots, \vec{a}_{N}\right\}$ denotes a vector subspace of $C^{N}$ spanned by $\vec{a}_{r+1}, \ldots$, $\vec{a}_{N-1}$ and $\vec{a}_{N}$.

Now we choose some vectors $\left\{\vec{l}_{i 2}(x)\right\}_{i=2}^{r}(C K(x))$ and $\left\{\vec{a}_{i 2}(x)\right\}_{i=r+2}^{N}$ so that $\left\{\vec{l}_{i 2}(x)\right\}_{i=1}^{r}$ is a base of $K(x)$ and $\left\{\vec{a}_{r+1,2}, \ldots, \vec{a}_{N 2}\right\}=\left\{\vec{a}_{r+1}, \ldots, \vec{a}_{N}\right\}$. Let us define $\left\{\vec{b}_{i 2}(x)\right\}_{i=1}^{N}$ as follows

$$
\begin{aligned}
& { }^{t} B_{1}(0, x) \vec{a}_{r+1,2}(x)=\vec{b}_{12}(x), \\
& { }^{t} B_{1}(0, x) \vec{l}_{i 2}(x)=\vec{b}_{i 2}(x), \quad(i=2, \cdots, r), \\
& { }^{t} A(0, x) \vec{a}_{i 2}(x)=\vec{b}_{i 2}(x), \quad(i=r+1, \ldots, N) .
\end{aligned}
$$

Hereafter we assume

(3. Ass.)

$$
{ }^{t} B_{k}(0, x) \vec{l}_{12}(x) / V(x)=0, \quad(k=2, \cdots, n) .
$$

Then we have

\section{Proposition 3.1.}

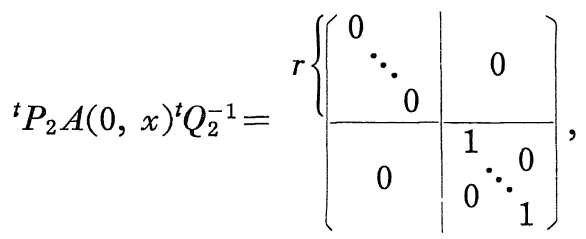

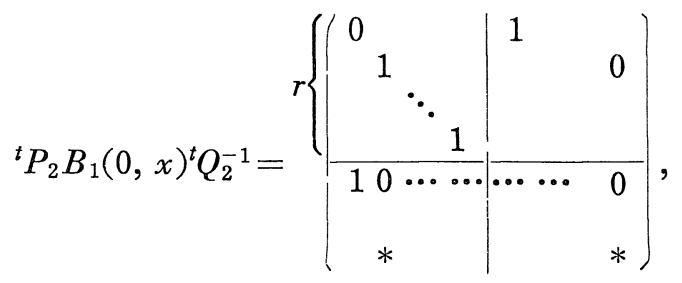




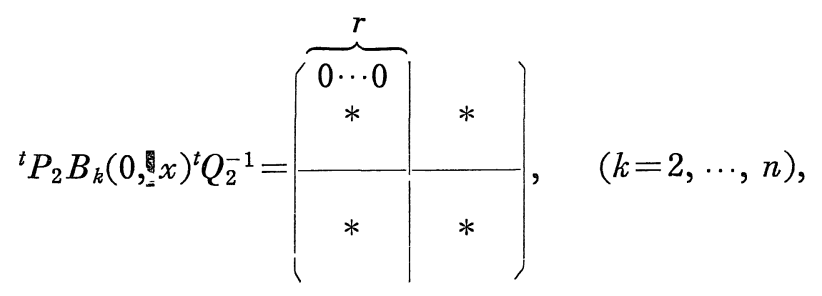

where $P_{2}(x)=\left(\vec{l}_{12}(x), \ldots, \vec{l}_{r 2}(x), \vec{a}_{r+1,2}(x), \ldots, \vec{a}_{N 2}(x)\right)$ and $Q_{2}(x)=\left(\vec{b}_{12}(x)\right.$, $\left.\cdots, \vec{b}_{N 2}(x)\right)$.

Proof. It suffices to prove that $\left\{\vec{b}_{i 2}(x)\right\}_{i=1}^{N}$ is a base of $C^{N}$. It is obvious that $\left\{\vec{b}_{i 2}(x)\right\}_{i=2}^{N}$ are linearly independent according to (3.1). If $\vec{b}_{12}(0) \in\left\{\vec{b}_{22}(0), \ldots, \vec{b}_{N 2}(0)\right\}$, we choose a vector $\vec{b}_{14}$ so that $\left\{\vec{b}_{14}, \vec{b}_{22}(0), \ldots\right.$, $\left.\vec{b}_{N 2}(0)\right\}$ is a base of $C^{N}$, then we have

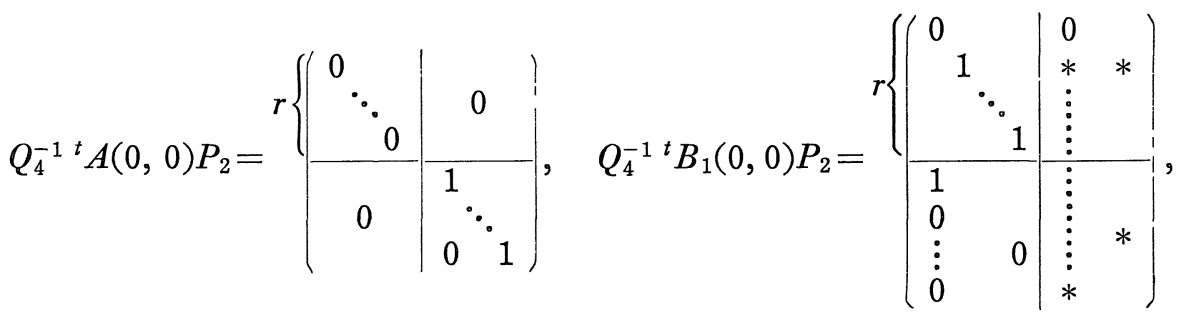

where $Q_{4}=\left(\vec{b}_{14}, \vec{b}_{22}(0), \ldots, \vec{b}_{N 2}(0)\right)$.

Now we define a modified characteristic polynomial $L_{2}(\tau, \xi)$ by

$$
\begin{aligned}
L_{2}(\tau, \xi) & \equiv \operatorname{det}\left(Q_{4}^{-1}\left({ }^{t} A(0,0) \tau+\sum_{k=1}^{n}{ }^{t} B_{k}(0,0) \xi_{k}\right) P_{2}\right) \\
& =\frac{\operatorname{det} P_{2}}{\operatorname{det} Q_{4}} H(0,0 ; \tau, \xi) .
\end{aligned}
$$

Then we have that the coefficient of $\xi_{1}^{r+1} \tau^{N-r-1}$ of $L_{2}(\tau, \xi)$ is zero. This contradicts $(1.4)^{\prime}$.

Q.E.D.

Now we transform the unknown function $\vec{u}$ to $\vec{v}$ by

$$
\vec{v}(t, x)={ }^{t} Q_{2}(x) \vec{u}(t, x),
$$

then we have the following system equivalent to (1.1); 


$$
\begin{aligned}
\{t a(t, x) & \left.\frac{\partial}{\partial t}+\sum_{k=1}^{n} t b_{k}(t, x) \frac{\partial}{\partial x_{k}}+c(t, x)\right\} v_{1}+\frac{\partial}{\partial x_{1}} v_{r+1} \\
= & \sum_{j=2}^{r} b_{1 j}\left(t, x ; t \frac{\partial}{\partial t}, t \frac{\partial}{\partial x_{1}}, t \frac{\partial}{\partial x^{\prime}}\right) v_{j} \\
& +\sum_{j=r+1}^{N} b_{1 j}\left(t, x ; t \frac{\partial}{\partial t}, t \frac{\partial}{\partial x_{1}}, \frac{\partial}{\partial x^{\prime}}\right) v_{j}+\left\langle\vec{l}_{12}, \vec{f}\right\rangle
\end{aligned}
$$

$$
\begin{array}{r}
\frac{\partial}{\partial x_{1}} v_{i}=\sum_{j=1}^{N} b_{i j}\left(t, x ; t \frac{\partial}{\partial t}, t \frac{\partial}{\partial x_{1}}, \frac{\partial}{\partial x^{\prime}}\right) v_{j}+<\vec{l}_{i 2}, \vec{f}>, \\
\quad(i=2, \ldots, r), \\
\frac{\partial}{\partial t} v_{r+1}+\frac{\partial}{\partial x_{1}} v_{1}=\sum_{j=1}^{N} b_{r+1, j}\left(t, x ; t \frac{\partial}{\partial t}, t \frac{\partial}{\partial x_{1}}, \frac{\partial}{\partial x^{\prime}}\right) v_{j} \\
+<\vec{a}_{r+1,2}, \vec{f}>, \\
\frac{\partial}{\partial t} v_{i}=\sum_{j=1}^{N} b_{i j}\left(t, x ; t \frac{\partial}{\partial t}, \frac{\partial}{\partial x_{1}}, \frac{\partial}{\partial x^{\prime}}\right) v_{j}+<\vec{a}_{i 2}, \vec{f}>, \\
\quad(i=r+2, \ldots, N),
\end{array}
$$

where $b_{i j}\left(t, x ; t \tau, t \xi_{1}, \xi^{\prime}\right)$ denote polynomials in $\tau, \xi_{1}$ and $\xi^{\prime}=\left(\xi_{2}, \ldots, \xi_{n}\right)$ of degree 1 .

Finally we note how to be deduced the functions $a(t, x)$ and $c(t, x)$ in the first equation of the above system from (1.1). Let $N(x)\left(C C^{N}\right)$ be the kernel of $A(0, x)$ and $W(x)$ be the image of $A(0, x)$, then we have $\operatorname{dim} B_{1}(0, x)(N(x)) / W(x)=r-1$. Then there exists a vector $\vec{r}(x) \in$ $N(x)$ such that

$$
B_{1}(0, x) \vec{r}(x) \in W(x), \quad B_{1}(0, x) \vec{r}(x) \neq 0 .
$$

It follows that $\left\langle\vec{b}_{i 2}(x), \vec{r}(x)\right\rangle=0,(i=2, \ldots, N)$ and $<\vec{l}_{12}(x)$, $B_{k}(0, x) \vec{r}(x)>=0,(k=2, \cdots, n) .^{3)}$

Then we have

Proposition 3.2. Under the assumption (1.4)' we have

3) $<\vec{l}_{12}(x), B_{k}(0, x) \vec{r}(x)>=0,(k=2, \ldots, n)$ follows from the assumption $(1.4)^{\prime}$. 


$$
\begin{aligned}
& <\vec{l}_{12}(x), \frac{\partial A}{\partial t} \vec{r}(x)>\left.\right|_{t=0}=a(0, x)<\vec{b}_{12}(x), \vec{r}(x)>, \\
& <\vec{l}_{12}(x), \mathscr{L}\left(0, x ; \frac{\partial}{\partial x}\right) \vec{r}(x)>=c(0, x)<\vec{b}_{12}(x), \vec{r}(x)>,
\end{aligned}
$$

where $\left\langle\vec{b}_{12}(x), \vec{r}(x)>\neq 0\right.$.

Proof. It is obvious according to the above reasonings.

\section{§4. Initial-value Problems in the Case Where $r \geqq 2$}

We now assume

$$
\frac{\partial^{t} A}{\partial t}(0, x) \vec{l}_{12}(x) / V(x)=0, \quad{ }^{t} \mathscr{L}\left(0, x ; \frac{\partial}{\partial x}\right) \vec{l}_{12}(x) / V(x)=0
$$

where ${ }^{t} \mathscr{L}\left(t, x ; \frac{\partial}{\partial x}\right) \vec{l}_{12}=-\sum_{k=1}^{n} \frac{\partial}{\partial x_{k}}\left({ }^{t} B_{k} \vec{l}_{12}\right)+{ }^{t} C \vec{l}_{12}$. We note that the assumption (4.1) implies

$$
\begin{aligned}
& a(t, x)=t \tilde{a}(t, x), \quad c(t, x)=t \tilde{c}(t, x), \\
& b_{1 j}\left(t, x ; t \frac{\partial}{\partial t}, t \frac{\partial}{\partial x_{1}}, t \frac{\partial}{\partial x^{\prime}}\right)=t \tilde{b}_{1 j}\left(t, x ; t \frac{\partial}{\partial t}, \frac{\partial}{\partial x_{1}}, \frac{\partial}{\partial x^{\prime}}\right),
\end{aligned}
$$

for any $j \in\{2, \ldots, r\}$ in the system (3.6). Then we have

Lemma 4.1. Under the assumption (4.1), the following boundaryvalue problem to the system (3.6) has a unique solution in a neighborhood of the origin:

$$
\begin{array}{ll}
\left.\left(\frac{\partial}{\partial x_{1}}\right)^{j} v_{1}\right|_{x_{1}=0}=W_{j}\left(t, x^{\prime}\right), & (j=0,1) \\
\left.v_{i}\right|_{x_{1}=0}=V_{i}\left(t, x^{\prime}\right), & (i=2, \cdots, r) \\
\left.v_{r+1}\right|_{x_{1}=t=0}=V_{r+1}\left(x^{\prime}\right), & \\
\left.v_{i}\right|_{t=0}=V_{i}(x), & (i=r+2, \ldots, N) .
\end{array}
$$

Theorem 4.1. (Initial-value problem) Under the assumptions in 
Lemma 4.1, the following conditions are necessary and also sufficient for the existence of a solution in a neighborhood of the origin of the initialvalue problem (1.1)-(1.2):

$$
\begin{aligned}
<\vec{l}_{i 2}(x), \mathscr{L}\left(0, x ; \frac{\partial}{\partial x}\right) \vec{U}(x)-\vec{f}(0, x)>=0, \quad(i=1, \ldots, r), \\
<\vec{l}_{12}(x),\left(A^{(1)}(x)+\mathscr{L}\left(0, x ; \frac{\partial}{\partial x}\right)\right) \vec{u}^{(1)}(x) \\
+\mathscr{L}^{(1)}\left(x ; \frac{\partial}{\partial x}\right) \vec{U}(x)-\vec{f}^{(1)}(x)>=0,
\end{aligned}
$$

where $A^{(1)}(x)=\frac{\partial A}{\partial t}(0, x), \mathscr{L}\left(t, x ; \frac{\partial}{\partial x}\right)=\sum_{k \geqq 0} t^{k} \mathscr{L}^{(k)}\left(x ; \frac{\partial}{\partial x}\right), \vec{f}(t, x)=\sum_{p \geqq 0}$ $\vec{f}^{(p)}(x) t^{p} / p$ ! and $\vec{u}^{(1)}(x)$ is a special solution of the equation

$$
A(0, x) \vec{u}^{(1)}(x)+\mathscr{L}\left(0, x ; \frac{\partial}{\partial x}\right) \vec{U}(x)=\vec{f}(0, x) .
$$

In Theorem 4.1, the necessity follows immediately from the construction of the formal solution of the problem (1.1)-(1.2),

$$
\vec{u}(t, x)=\sum_{p \geqq 0} \vec{u}^{(p)}(x) t^{p} / p ! .
$$

Lemma 4.2. (Goursat-problem) Let us assume

$$
a(0, x) \neq 0, p a(0, x)+c(0, x) \neq 0 \quad \text { for } p \in\{0,1,2, \ldots\} .
$$

Then the following Goursat-problem to the system (3.6) has a unique solution in a neighborhood of the origin:

$$
\begin{array}{ll}
\left.v_{i}\right|_{x_{1}=0}=V_{i}\left(t, x^{\prime}\right), & (i=2, \cdots, r), \\
\left.v_{i}\right|_{t=0}=V_{i}(x), & (i=r+1, \ldots, N) .
\end{array}
$$

Theorem 4.2. (Initial-value problem) Under the assumptions in Lemma 4.2, the following compatibility conditions are necessary and sufficient in order to exist at least one solution of the initial-value problem (1.1) -(1.2) in a neighborhood of the origin:

$$
<\vec{l}_{i 2}(x), \mathscr{L}\left(0, x ; \frac{\partial}{\partial x}\right) \vec{U}(x)-\vec{f}(0, x)>=0, \quad(i=1, \ldots, r) .
$$


In Lemma 4.2 we can not eliminate the condition $a(0, x) \neq 0$. In fact if we assume

$$
a(0, x) \equiv 0, c(0, x) \neq 0
$$

we have

Theorem 4.3. (Goursat-problem) Let us assume (4.8), then the Goursat-problem (3.6)-(4.6) has not always an analytic solution in a neighborhood of the origin. Precisely we can find such Goursat-data that there exists no analytic solution in any neighborhood of the origin.

Remark 4.1. In Theorem 4.1, if we only assume $a(0, x) \equiv c(0, x) \equiv$ 0 , we can not obtain necessary and sufficient conditions of type (4.3). Moreover, without the condition (3. Ass.), we can not obtain the compatibility conditions of type (4.3) for the existence of solutions of problem (1.1)-(1.2) (see Theorem A.1).

Remark 4.2. Under the assumptions in Theorem 4.3, we can not decide whether the initial-value problem (1.1)-(1.2) has a solution or not in view of Theorem 4.3.

Remark 4.3. In the case where $a(0, x) \neq 0, p_{0} a(0, x)+c(0, x) \equiv 0$ for some $p_{0} \in\{0,1,2, \ldots\}$, there can not exist a solution of the Goursatproblem (3.6)-(4.6) without compatibility conditions between the Goursatdata (4.6). Moreover even if there exists a solution, it is not unique in general.

Remark 4.4. The following Goursat-problem to the system (3.6) has a unique solution in a neighborhood of the origin;

$$
\begin{array}{ll}
\left.v_{i}\right|_{x_{1}=0}=V_{i}\left(t, x^{\prime}\right), & (i=1, \ldots, r+1), \\
\left.v_{i}\right|_{t=0}=V_{i}(x), & (i=r+2, \ldots, N) .
\end{array}
$$

The proof is similar to that of Lemma 4.2 , or see Théorème 3.1 in [2]. From this Goursat-problem we see that we can give data of $v_{i}, \quad(i=r+$ $2, \ldots, N)$ at $t=0$ arbitrarily. 


\section{§5. Proof of Theorem 4.1}

Proposition 5.1. ${ }^{4)}$ Let us consider the following Goursat-problem, then there exists a unique solution in a neighborhood of the origin:

$$
\begin{gathered}
\frac{\partial^{2}}{\partial x_{1}^{2}} v_{1}=\sum_{j=1}^{N}\left\{t a_{1 j}(t, x) \frac{\partial^{2}}{\partial t^{2}}+t b_{1 j}(t, x) \frac{\partial^{2}}{\partial t \partial x_{1}}+t c_{1 j}(t, x) \frac{\partial^{2}}{\partial x_{1}^{2}}\right. \\
\left.+d_{1 j}\left(t, x ; \frac{\partial}{\partial t}, \frac{\partial}{\partial x_{1}}, \frac{\partial}{\partial x^{\prime}}\right)\right\} v_{j}+h_{1}(t, x),
\end{gathered}
$$

$$
\begin{array}{cc}
\frac{\partial}{\partial x_{1}} v_{i}=\sum_{j=1}^{N}\left\{t a_{i j}(t, x) \frac{\partial}{\partial t}+t b_{i j}(t, x) \frac{\partial}{\partial x_{1}}+c_{i j}\left(t, x ; \frac{\partial}{\partial x^{\prime}}\right)\right\} v_{j} \\
+h_{i}(t, x), & (i=2, \ldots, r), \\
\frac{\partial}{\partial t} v_{i}=\sum_{j=1}^{N}\left\{t a_{i j}(t, x) \frac{\partial}{\partial t}+b_{i j}\left(t, x ; \frac{\partial}{\partial x_{1}}, \frac{\partial}{\partial x^{\prime}}\right)\right\} v_{j}+h_{i}(t, x), & (i=r+1, \ldots, N) . \\
\left.\left(\frac{\partial}{\partial x_{1}}\right)^{j} v_{1}\right|_{x_{1}=0}=W_{j}\left(t, x^{\prime}\right), & (j=0,1), \\
\left.v_{i}\right|_{x_{1}=0}=V_{i}\left(t, x^{\prime}\right), & (i=2, \cdots, r), \\
\left.v_{i}\right|_{t=0}=V_{i}(x), & (i=r+1, \ldots, N),
\end{array}
$$

where $b_{i j}$ and $c_{i j}$ are differential operators of the first order, and $d_{1 j}$ are differential operators of the second order in which $\frac{\partial^{2}}{\partial t^{2}}, \frac{\partial^{2}}{\partial t \partial x_{1}}$ and $\frac{\partial^{2}}{\partial x_{1}^{2}}$ do not appear.

Remark 5.1. The system (5.1) is obtained as follows; differentiating the first equation in (3.6) with respect to $t$ and substituting the term $\frac{\partial}{\partial t} v_{r+1}$ by the third equation in (3.6).

Proof. Without loss of generality we may assume that the Goursatdata are all zero. We can easily show that the formal solution of type

4) A more general Goursat-problem was treated by L. Gårding ([2], Théorème 3.2). We here give a more elementary proof. 


$$
v_{i}(t, x)=\sum_{p, q \geqq 0} v_{i}^{(p, q)}\left(x^{\prime}\right) t^{p} x_{1}^{q} / p ! q !, \quad(i=1,2, \cdots, N)
$$

can be constructed uniquely.

In order to prove the convergence of the formal solution in a neighborhood of the origin, we make a majorant system of (5.1).

Let

$$
X=\frac{t}{\alpha}+x_{1}, \quad Y=x_{2}+\cdots+x_{n}
$$

where $\alpha$ is a sufficiently small positive constant defined later. Let $M /$ $\left(1-\frac{X+Y}{\rho}\right)$ be a common majorant of $a_{i j}(t, x), \ldots$ and the coefficients of $d_{1 j}\left(t, x ; \frac{\partial}{\partial t}, \frac{\partial}{\partial x_{1}}, \frac{\partial}{\partial x^{\prime}}\right)$, and let $C M N /\left(1-\frac{X+Y}{\rho}\right)$ be a majorant of $h_{i}(t, x),(i=1, \ldots, N)$.

Then we have the following majorant system of (5.1):

$$
\begin{aligned}
\frac{\partial^{2}}{\partial x_{1}^{2}} w_{1}=\frac{M N}{1-\frac{X+Y}{\rho}\left\{\sum_{j=1}^{3}\right.}( & X \frac{\partial^{2}}{\partial t^{2}}+X \frac{\partial^{2}}{\partial t \partial x_{1}}+X \frac{\partial^{2}}{\partial x_{1}^{2}} \\
& \left.\left.+d_{1 j}\left(\frac{\partial}{\partial t}, \frac{\partial}{\partial x_{1}}, \frac{\partial}{\partial x_{2}}, \cdots, \frac{\partial}{\partial x_{n}}\right)\right) w_{j}+C\right\},
\end{aligned}
$$

$$
\begin{aligned}
& \frac{\partial}{\partial x_{1}} w_{2}=\frac{M N}{1-\frac{X+Y}{\rho}}\left\{\sum_{j=1}^{3}\left(X \frac{\partial}{\partial t}+X \frac{\partial}{\partial x_{1}}+\tilde{b}_{2 j}\left(\frac{\partial}{\partial x_{2}}, \cdots, \frac{\partial}{\partial x_{n}}\right)\right) w_{j}+C\right\}, \\
& \frac{\partial}{\partial t} w_{3}=\frac{M N}{1-\frac{X+Y}{\rho}}\left\{\sum_{j=1}^{3}\left(X \frac{\partial}{\partial t}+\frac{\partial}{\partial x_{1}}+\tilde{b}_{3 j}\left(\frac{\partial}{\partial x_{2}}, \cdots, \frac{\partial}{\partial x_{n}}\right)\right) w_{j}+C\right\},
\end{aligned}
$$

where $\tilde{b}_{i j}$ are differential operators with positive coefficients of the first order, and $\tilde{d}_{1 j}$ are differential operators with positive coefficients of the second order which do not contain the terms $\frac{\partial^{2}}{\partial t^{2}}, \frac{\partial^{2}}{\partial t \partial x_{1}}$ and $\frac{\partial^{2}}{\partial x_{1}^{2}}$.

We shall prove the existence of a solution of (5.4) with non-negative coefficients. Clearly if such a solution exists, it is a majorant of the formal solution (5.3).

Now let us construct a solution of (5.4) depending on $X$ and $Y$. 
Namely, let us consider the following initial-value problem:

$$
\begin{aligned}
& \left(1-\frac{X+Y}{\rho}\right) \frac{\partial^{2}}{\partial X^{2}} w_{1}=M N\left[\sum _ { j = 1 } ^ { 3 } \left\{\left(\frac{1}{\alpha^{2}}+\frac{1}{\alpha}+1\right) X \frac{\partial^{2}}{\partial X^{2}}\right.\right. \\
& \left.\left.+\tilde{d}_{1 j}\left(\frac{1}{\alpha} \frac{\partial}{\partial X}, \frac{\partial}{\partial X}, \frac{\partial}{\partial Y}, \cdots, \frac{\partial}{\partial Y}\right)\right\} w_{j}+C\right] \\
& \left(1-\frac{X+Y}{\rho}\right) \frac{\partial}{\partial X} w_{2}=M N\left[\sum _ { j = 1 } ^ { 3 } \left\{\left(\frac{1}{\alpha}+1\right) X \frac{\partial}{\partial X}\right.\right. \\
& \left.\left.+\tilde{b}_{2 j}\left(\frac{\partial}{\partial Y}, \cdots, \frac{\partial}{\partial Y}\right)\right\} w_{j}+C\right] \\
& \left\{\left(\frac{1}{\alpha}-M N\right)-\frac{X+Y}{\alpha \rho}\right\} \frac{\partial}{\partial X} w_{3}-M N \frac{\partial}{\partial X} w_{1}-M N \frac{\partial}{\partial X} w_{2} \\
& =M N\left[\sum_{j=1}^{3}\left(\frac{X}{\alpha} \frac{\partial}{\partial X}+\tilde{b}_{3 j}\left(\frac{\partial}{\partial Y}, \cdots, \frac{\partial}{\partial Y}\right)\right) w_{j}+C\right] \\
& \left.\left(\frac{\partial}{\partial X}\right)^{j} w_{1}\right|_{X=0}=0, \quad(j=0,1),\left.\quad w_{j}\right|_{X=0}=0, \quad(j=2,3) .
\end{aligned}
$$

If we choose sufficiently small $\alpha$, the formal solution of (5.5)-(5.6),

$$
w_{i}(X, Y)=\sum_{p, q \geqq 0} w_{i}^{(p, q)} X^{p} Y^{q} / p ! q !, \quad(i=1,2,3)
$$

has non-negative coefficients. In fact it suffices to choose $\alpha$ so that $0<$ $\alpha<1 / M N$.

In order to prove the convergence of (5.7) in a neighborhood of the origin, we reduce the problem (5.5)-(5.6) to the equivalent problem as follows; differentiating the second and the third equations with respect to $X$, then we have a second order system. Now we give the following data $\left.\frac{\partial w_{j}}{\partial X}\right|_{X=0},(j=2,3)$;

$$
\begin{aligned}
& \left.\frac{\partial w_{2}}{\partial X}\right|_{X=0}=\left(1-\frac{Y}{\rho}\right)^{-1} C M N \\
& \left.\frac{\partial w_{3}}{\partial X}\right|_{X=0}=\left(\frac{1}{\alpha}-M N-\frac{Y}{\alpha \rho}\right)^{-1}\left\{\left.M N \frac{\partial w_{2}}{\partial X}\right|_{X=0}+C M N\right\},
\end{aligned}
$$

in a neighborhood of $Y=0$.

It is obvious that the initial-value problem obtained by the above 
procedure has a unique solution in a neighborhood of the origin. This completes the proof.

Q.E.D.

Proof of Lemma 4.1. It is sufficient to prove that the problem (3.6)(4.2) can be reduced to the Goursat-problem considered in Proposition 5.1 under the assumption $(4.1)^{\prime}$.

We remark that under the assumption $(4.1)^{\prime}$ we can construct the formal solution of the problem (3.6)-(4.2) of type

$$
v_{i}(t, x)=\sum_{p \geqq 0} v_{i}^{(p)}(x) t^{p} / p !, \quad(i=1, \cdots, N),
$$

instead of the formal solution of type (5.3).

It is easy to see that $v_{r+1}^{(0)}(x)$ should be a solution of the following initial-value problem;

$$
\begin{gathered}
\frac{\partial}{\partial x_{1}} v_{r+1}^{(0)}=\sum_{j=r+1}^{N} b_{1 j}\left(0, x ; 0,0, \frac{\partial}{\partial x^{\prime}}\right) v_{j}^{(0)}(x)+<\vec{l}_{12}, \vec{f}((0, x)>, \\
\left.v_{r+1}^{(0)}\right|_{x_{1}=0}=V_{r+1}\left(x^{\prime}\right),
\end{gathered}
$$

where $v_{j}^{(0)}(x)=V_{j}(x),(j=r+2, \ldots, N)$.

Now let us consider the following. Goursat-problem equivalent to the problem (3.6)-(4.2); differentiating the first equation in (3.6) with respect to $t$ and substituting the terms $\frac{\partial}{\partial t} v_{i},(i=r+1, \ldots, N)$ by the third and the fourth equations in (3.6), then we have the following system

$$
\begin{aligned}
& \frac{\partial^{2}}{\partial x_{1}^{2}} v_{1}= \sum_{j=1}^{N}\left\{t^{2} \tilde{a}_{1 j}(t, x) \frac{\partial^{2}}{\partial t^{2}}+t \tilde{b}_{1 j}\left(t, x ; \frac{\partial}{\partial x_{1}}, \frac{\partial}{\partial x^{\prime}}\right) \frac{\partial}{\partial t}\right. \\
&\left.+t \tilde{c}_{1 j}(t, x) \frac{\partial^{2}}{\partial x_{1}^{2}}+\tilde{d}_{1 j}\left(t, x ; \frac{\partial}{\partial x_{1}}, \frac{\partial}{\partial x^{\prime}}\right)\right\} v_{j}+h_{1}(t, x), \\
& \frac{\partial}{\partial x_{1}} v_{i}=\sum_{j=1}^{N} b_{i j}\left(t, x ; t \frac{\partial}{\partial t}, t \frac{\partial}{\partial x_{1}}, \frac{\partial}{\partial x^{\prime}}\right) v_{j}+<\vec{l}_{i 2}, \vec{f}>, \\
& \quad(i=2, \ldots, r), \\
& \frac{\partial}{\partial t} v_{i}=\sum_{j=1}^{N} b_{i j}\left(t, x ; t \frac{\partial}{\partial t}, \frac{\partial}{\partial x_{1}}, \frac{\partial}{\partial x^{\prime}}\right) v_{j}+<\vec{l}_{i 2}, \vec{f}>, \\
& \quad(i=r+1, \ldots, N),
\end{aligned}
$$


where $\tilde{b}_{i j}$ are differential operators of the first order, and $\tilde{d}_{1 j}$ are differential operators of the second order in which $\frac{\partial^{2}}{\partial x_{1}^{2}}$ does not appear.

Goursat-data are given by

$$
\begin{array}{ll}
\left.\left(\frac{\partial}{\partial x_{1}}\right)^{j} v_{1}\right|_{x_{1}=0}=W_{j}\left(t, x^{\prime}\right), & (j=0,1), \\
\left.v_{i}\right|_{x_{1}=0}=V_{i}\left(t, x^{\prime}\right), & (i=2, \ldots, r), \\
\left.v_{r+1}\right|_{t=0}=v_{r+1}^{(0)},\left.v_{i}\right|_{t=0}=V_{i}(x), & (i=r+2, \ldots, N) .
\end{array}
$$

Clearly the problem (5.12)-(5.13) has the same formal solution with that of (3.6)-(4.2). The problem (5.12)-(5.13) is a special case considered in Proposition 5.1, then there exists a unique solution in a neighborhood of the origin. This completes the proof.

Before we prove Theorem 4.1, we note that $v_{i}^{(0)}(x),(i=1, \ldots, r)$ should be a solution of the initial-value problem

$$
\begin{aligned}
& \frac{\partial^{2}}{\partial x_{1}^{2}} v_{1}^{(0)}=\sum_{j=1}^{N} \tilde{d}_{1 j}\left(0, x ; \frac{\partial}{\partial x_{1}}, \frac{\partial}{\partial x^{\prime}}\right) v_{j}^{(0)}+h_{1}(0, x), \\
& \frac{\partial}{\partial x_{1}} v_{i}^{(0)}=\sum_{j=1}^{N} b_{i j}\left(0, x ; 0,0, \frac{\partial}{\partial x^{\prime}}\right) v_{j}^{(0)}+<\vec{l}_{i 2}, \vec{f}(0, x)>, \\
& (i=2, \ldots, r), \\
& \left.\left(\frac{\partial}{\partial x_{1}}\right)^{j} v_{1}^{(0)}\right|_{x_{1}=0}=W_{j}\left(0, x^{\prime}\right), \quad(j=0,1), \\
& \left.v_{i}^{(0)}\right|_{x_{1}=0}=V_{i}\left(0, x^{\prime}\right), \quad(i=2, \ldots, r) .
\end{aligned}
$$

Proof of Theorem 4.1. We shall prove the theorem to the system (3.6) instead of the problem (1.1)-(1.2). Then the compatibility condition (4.3) is transformed into (5.10) and (5.14).

Since the necessity is obvious, we prove the sufficiency. Now let us consider the following Goursat-problem to (3.6);

$$
\left.\left(\frac{\partial}{\partial x_{1}}\right)^{j} v_{1}\right|_{x_{1}=0}=\left.\left(\frac{\partial}{\partial x_{1}}\right)^{j} V_{1}(x)\right|_{x_{1}=0}+t x^{(j)}\left(t, x^{\prime}\right), \quad(j=0,1)
$$




$$
\begin{aligned}
& \left.v_{i}\right|_{x_{1}=0}=V_{i}\left(0, x^{\prime}\right)+t x_{i}\left(t, x^{\prime}\right), \quad(i=2, \cdots, r), \\
& \left.v_{r+1}\right|_{t=x_{1}=0}=V_{r+1}\left(0, x^{\prime}\right),\left.v_{i}\right|_{t=0}=V_{i}(x), \quad(i=r+2, \ldots, N),
\end{aligned}
$$

where $\vec{V}(x)={ }^{t}\left(V_{1}(x), \ldots, V_{N}(x)\right)$ is the initial-data of (3.6), and $x^{(j)}$ and $x_{i}$ are arbitrary analytic functions in $t$ and $x^{\prime}$.

Then there exists a unique solution $v_{i}(t, x),(i=1,2, \ldots, N)$ of the problem (3.6)-(5.16) in a neighborhood of the origin. This solution is also a solution of the initial-value problem to the system (3.6). In fact, it suffices to show $v_{i}^{(0)}(x)=V_{i}(x),(i=1, \ldots, r+1)$. It follows from the construction of the Goursat-data (5.16) and the compatibility conditions (5.10) and (5.14).

The non-uniqueness of the solution is obvious from the arbitrariness of $\chi^{(j)}$ and $\chi_{i}$ in (5.16). This completes the proof.

Q.E.D.

\section{§6. Proof of Theorem 4.2}

Lemma 6.1. (Goursat-problem) Let us consider the following Goursat-problem, then there exists a unique solution in a neighborhood of the origin:

$$
\begin{aligned}
\left\{t\left(\frac{\partial}{\partial t}\right)^{2}+\frac{\partial}{\partial t}\right\} v_{1}= & M_{1}\left(x^{\prime}\right)\left[\left\{t^{2}\left(\frac{\partial}{\partial t}\right)^{2}+t x_{1}\left(\frac{\partial}{\partial t}\right)^{2}\right.\right. \\
& \left.+\sum_{k=1}^{n} t \frac{\partial^{2}}{\partial t \partial x_{k}}+t \frac{\partial}{\partial t}+x_{1} \frac{\partial}{\partial t}\right\} v_{1} \\
& +\sum_{2 \leqq j \leqq 3}\left\{t\left(\frac{\partial}{\partial t}\right)^{2}+\sum_{k=1}^{n} t \frac{\partial^{2}}{\partial t \partial x_{k}}+\frac{\partial}{\partial t}\right\} v_{j} \\
& \left.+\sum_{\substack{1 \leqq j \leqq 3 \\
l+|\nu| \leqq 2}}\left(\frac{\partial}{\partial x_{1}}\right)^{l}\left(\frac{\partial}{\partial x^{\prime}}\right)^{\nu} v_{j}\right]+F_{1}\left(x^{\prime}\right),
\end{aligned}
$$

$$
\begin{gathered}
\frac{\partial^{2}}{\partial t \partial x_{1}} v_{2}=M_{2}\left(x^{\prime}\right)\left[x_{1} \frac{\partial^{2}}{\partial t \partial x_{1}} v_{2}+\sum_{\substack{1 \leqq j \leqq 3 \\
i+l=2}} t\left(\frac{\partial}{\partial t}\right)^{i}\left(\frac{\partial}{\partial x_{1}}\right)^{l} v_{j}\right. \\
\left.+\sum_{\substack{1 \leqq j \leqq 3 \\
i+l \leq l \leq 1 \\
i+l+|\nu| \leq 2}}\left(\frac{\partial}{\partial t}\right)^{i}\left(\frac{\partial}{\partial x_{1}}\right)^{l}\left(\frac{\partial}{\partial x^{\prime}}\right)^{\nu} v_{j}\right]+F_{2}\left(x^{\prime}\right),
\end{gathered}
$$




$$
\begin{aligned}
\frac{\partial^{2}}{\partial t^{2}} v_{3}=M_{3}\left(x^{\prime}\right)\left[x_{1} \frac{\partial^{2}}{\partial t^{2}} v_{3}+\sum_{1 \leqq j \leqq 3} t \frac{\partial^{2}}{\partial t^{2}} v_{j}\right. \\
\left.+\sum_{\substack{1 \leqq j \leqq 3 \\
i \leq 1 \\
i+l+|\nu| \leqq 2}}\left(\frac{\partial}{\partial t}\right)^{i}\left(\frac{\partial}{\partial x_{1}}\right)^{l}\left(\frac{\partial}{\partial x^{\prime}}\right)^{\nu} v_{j}\right]+F_{3}\left(x^{\prime}\right),
\end{aligned}
$$

$$
\left.v_{1}\right|_{t=0}=0,\left.\quad v_{2}\right|_{t=0}=\left.v_{2}\right|_{x_{1}=0}=0,\left.\quad\left(\frac{\partial}{\partial t}\right)^{j} v_{3}\right|_{t=0}=0, \quad(j=0,1)
$$

Remark 6.1. For the simplicity, we assumed that the coefficients in the right hand side are represented by $M_{i}\left(x^{\prime}\right)$, although the lemma is true if the coefficient of each differential appeared in the right hand side is a function depending on $x^{\prime}$.

In generally the lemma is true for the higher order system (see the proof in detail).

Proof. Let us construct the formal solution of type

$$
v_{i}(t, x)=\sum_{p, q \geqq 0} v_{i}^{(p, q)}\left(x^{\prime}\right) \frac{t^{p}}{p !} \frac{x_{1}^{q}}{q !}
$$

Substituting (6.3) into (6.1) and comparing the coefficients of $t^{p} x_{1}^{q}$, we have

$$
\begin{aligned}
(p+1) v_{1}^{(p+1, q)} & =M_{1}\left(x^{\prime}\right)\left[p^{2} v_{1}^{(p, q)}+(p+1) q v_{1}^{(p+1, q-1)}+p v_{1}^{(p, q+1)}\right. \\
& +\sum_{k=2}^{n} p \frac{\partial}{\partial x_{k}} v_{1}^{(p, q)}+\sum_{2 \leqq j \leqq 3}\left\{p v_{j}^{(p+1, q)}+p v_{j}^{(p, q+1)}\right. \\
& \left.\left.+\sum_{k=2}^{n} p \frac{\partial}{\partial x_{k}} v_{j}^{(p, q)}+v_{j}^{(p+1, q)}\right\}+\sum_{\substack{1 \leqq j \leq 3 \\
l+|\nu| \leq 2}}\left(\frac{\partial}{\partial x^{\prime}}\right)^{\nu} v_{j}^{(p, l+q)}\right] \\
& +F_{1}\left(x^{\prime}\right) \delta_{0}^{p} \delta_{0}^{q},
\end{aligned}
$$

$$
\begin{aligned}
v_{2}^{(p+1, q+1)} & =M_{2}\left(x^{\prime}\right)\left[q v_{2}^{(p+1, q)}+\sum_{\substack{1 \leqq j \leqq 3 \\
i+l=2}} p v_{j}^{(i+p-1, l+q)}\right. \\
& \left.+\sum_{\substack{1 \leqq j \leqq 3 \\
i \leq l \leq 1 \\
i+l+|\nu| \leqq 2}}\left(\frac{\partial}{\partial x^{\prime}}\right)^{\nu} v_{j}^{(i+p, l+q)}\right]+F_{2}\left(x^{\prime}\right) \delta_{0}^{p} \delta_{0}^{q}
\end{aligned}
$$




$$
\begin{aligned}
v_{3}^{(p+2, q)}= & M_{3}\left(x^{\prime}\right)\left[q v_{3}^{(p+2, q-1)}+\sum_{1 \leqq j \leqq 3} p v_{j}^{(p+1, q)}\right. \\
& \left.+\sum_{\substack{1 \leqq j \leqq 3 \\
i \leq 1 \\
i+l+|\nu| \leqq 2}}\left(\frac{\partial}{\partial x^{\prime}}\right)^{\nu} v_{j}^{(i+p, l+q)}\right]+F_{3}\left(x^{\prime}\right) \delta_{0}^{p} \delta_{0}^{q}
\end{aligned}
$$

where $\delta_{0}^{p}$ and $\delta_{0}^{q}$ are Kronecker's $\delta$.

Since $M_{i}\left(x^{\prime}\right)$ and $F_{i}\left(x^{\prime}\right)$ are analytic functions, we assume

$$
\left|\left(\frac{\partial}{\partial x^{\prime}}\right)^{\alpha} M_{i}\left(x^{\prime}\right)\right| \leqq \frac{|\alpha| !}{(3 \rho)^{|\alpha|}} A, \quad\left|\left(\frac{\partial}{\partial x^{\prime}}\right)^{\alpha} F_{i}\left(x^{\prime}\right)\right| \leqq \frac{|\alpha| !}{\rho^{|\alpha|}} B
$$

in $\left|x^{\prime}\right|<\delta$, for some positive constants $A, B, \rho$ and $\delta$.

Then we can prove the following estimates by induction on $p$ and $q$,

$$
\begin{aligned}
& \left|\left(\frac{\partial}{\partial x^{\prime}}\right)^{\alpha} v_{1}^{(p, q)}\left(x^{\prime}\right)\right| \leqq \frac{(2 p+q+|\alpha|) !}{p !} C^{7 p+3 q+1+|\alpha|} B, \\
& \left|\left(\frac{\partial}{\partial x^{\prime}}\right)^{\alpha} v_{i}^{(p, q)}\left(x^{\prime}\right)\right| \leqq \frac{(2 p+q+|\alpha|) !}{p !} C^{7 p+3 q+|\alpha|} B, \quad(i=2,3),
\end{aligned}
$$

for some positive constant $C$ depending on $A, \rho$ and the number of terms of summations in the right hand side of (6.1). It is easy to prove (6.6) by the lemma due to S. Mizohata [7]:

Let $a(x)$ and $b(x)$ be analytic functions satisfying

$$
\begin{aligned}
& \left|\left(\frac{\partial}{\partial x}\right)^{\alpha} a(x)\right| \leqq \frac{(r+|\alpha|) !}{(k \rho)^{|\alpha|}} A, \quad(k>1), \\
& \left|\left(\frac{\partial}{\partial x}\right)^{\alpha} b(x)\right| \leqq \frac{(s+|\alpha|) !}{\rho^{|\alpha|}} B
\end{aligned}
$$

where $r$ and $s$ are non-negative integers. Then we have

$$
\left|\left(\frac{\partial}{\partial x}\right)^{\alpha}\{a(x) b(x)\}\right| \leqq \frac{(r+s+|\alpha|) !}{\rho^{|\alpha|}} \frac{k}{k-1} A B / C_{r}^{r+s} .
$$

We omit to prove (6.6). (Similar estimates are given in [6].)

Then we have the following estimates for the formal solution

$$
\left|\left(\frac{\partial}{\partial t}\right)^{p}\left(\frac{\partial}{\partial x_{1}}\right)^{q}\left(\frac{\partial}{\partial x^{\prime}}\right)^{\alpha} v_{i}(t, x)\right| \leqq(p+q+|\alpha|) ! D^{p+q+|\alpha|} E
$$


$(i=1,2,3)$, in $|t|<\gamma,\left|x_{1}\right|<\gamma,\left|x^{\prime}\right|<\delta$ for some positive constants $D, E$ and $\gamma$. Thus we proved the lemma.

Q.E.D.

Proof of Lemma 4.2. Let us recall the assumption (4.5),

$$
a(0, x) \neq 0, \quad p a(0, x)+c(0, x) \neq 0,
$$

for any $p \in\{0,1,2, \ldots\}$, then we may assume $a(t, x)=1, p+c(0, x) \neq 0$.

Especially,

$$
p+c\left(0,0, x^{\prime}\right) \neq 0 \quad \text { for } p \in\{0,1,2, \ldots\} .
$$

Thus we may rewrite the system (3.6) as follows;

$$
\begin{aligned}
& \left\{t \frac{\partial}{\partial t}+c\left(0,0, x^{\prime}\right)\right\} v_{1}=\left\{\sum_{k=1}^{n} t b_{k}(t, x) \frac{\partial}{\partial x_{k}}+t \tilde{c}(t, x)+x_{1} \tilde{\widetilde{c}}(t, x)\right\} v_{1} \\
& \quad+\sum_{j=2}^{r} b_{1 j}\left(t, x ; t \frac{\partial}{\partial t}, t \frac{\partial}{\partial x_{1}}, t \frac{\partial}{\partial x^{\prime}}\right) v_{j} \\
& \quad+\sum_{j=r+1}^{N} b_{1 j}\left(t, x ; t \frac{\partial}{\partial t}, \frac{\partial}{\partial x_{1}}, \frac{\partial}{\partial x^{\prime}}\right) v_{j}+g_{1}(t, x), \\
& \frac{\partial}{\partial x_{1}} v_{i}=\sum_{j=1}^{N} b_{i j}\left(t, x ; t \frac{\partial}{\partial t}, t \frac{\partial}{\partial x_{1}}, \frac{\partial}{\partial x^{\prime}}\right) v_{j}+g_{i}(t, x), \quad(i=2, \ldots, r), \\
& \frac{\partial}{\partial t} v_{i}=\sum_{j=1}^{N} b_{i j}\left(t, x ; t \frac{\partial}{\partial t}, \frac{\partial}{\partial x_{1}}, \frac{\partial}{\partial x^{\prime}}\right) v_{j}+g_{i}(t, x), \quad(i=r+1, \ldots, N) .
\end{aligned}
$$

It is obvious that the formal solution of (6.9)-(4.6) of type

$$
v_{i}(t, x)=\sum_{p, q \geqq 0} v_{i}^{(p, q)}\left(x^{\prime}\right) \frac{t^{p}}{p !} \frac{x_{1}^{q}}{q !}, \quad(i=1,2, \ldots, N),
$$

can be constructed uniquely.

In order to prove the analyticity of (6.10) in a neighborhood of the origin, we consider the following Goursat-problem equivalent to (6.9)(4.6); differentiating the first equation in (6.9) with respect to $t$, and substituting the terms $\frac{\partial}{\partial t} v_{i},(i=r+1, \ldots, N)$ by the third equations in 
(6.9), we have

$$
\begin{aligned}
\left\{t \frac{\partial^{2}}{\partial t^{2}}\right. & \left.+\left(1+c\left(0,0, x^{\prime}\right)\right) \frac{\partial}{\partial t}\right\} v_{1}=\left\{\sum_{k=1}^{n} t \tilde{b}_{k}(t, x) \frac{\partial^{2}}{\partial x_{k} \partial t}+t \tilde{e}(t, x) \frac{\partial}{\partial t}\right. \\
& \left.+x_{1} \tilde{e}(t, x) \frac{\partial}{\partial t}+\tilde{d}_{11}\left(t, x ; \frac{\partial}{\partial x_{1}}, \frac{\partial}{\partial x^{\prime}}\right)\right\} v_{1} \\
& +\sum_{j=2}^{N}\left\{t a_{j}(t, x) \frac{\partial^{2}}{\partial t^{2}}+\sum_{k=1}^{n} t b_{k j}(t, x) \frac{\partial^{2}}{\partial x_{k} \partial t}\right. \\
& \left.+\tilde{d}_{1 j}\left(t, x ; \frac{\partial}{\partial t}, \frac{\partial}{\partial x_{1}}, \frac{\partial}{\partial x^{\prime}}\right)\right\} v_{j}+h_{1}(t, x),
\end{aligned}
$$

$$
\begin{aligned}
& \frac{\partial^{2}}{\partial x_{1} \partial t} v_{i}=\frac{\partial}{\partial t}\left\{\sum_{j=1}^{N} b_{i j}\left(t, x ; t \frac{\partial}{\partial t}, t \frac{\partial}{\partial x_{1}}, \frac{\partial}{\partial x^{\prime}}\right) v_{j}+g_{i}\right\}, \\
& \quad(i=2, \ldots, r), \\
& \frac{\partial^{2}}{\partial t^{2}} v_{i}=\frac{\partial}{\partial t}\left\{\sum_{j=1}^{N} b_{i j}\left(t, x ; t \frac{\partial}{\partial t}, \frac{\partial}{\partial x_{1}}, \frac{\partial}{\partial x^{\prime}}\right) v_{j}+g_{i}\right\},
\end{aligned}
$$$$
(i=r+1, \ldots, N)
$$

where $\tilde{d}_{1 j},(j=1,2, \ldots, N)$ are differential operators of the second order, and $\tilde{d}_{1 j},(j=2, \ldots, N)$ do not involve terms $\frac{\partial^{2}}{\partial t^{2}}$ and $\frac{\partial^{2}}{\partial x_{k} \partial t},(k=1, \ldots, n)$. Goursat-data of (6.11) are given by

$$
\begin{aligned}
& \left.V_{1}\right|_{t-0}=v_{1}^{(0)}(x), \\
& \left.v_{i}\right|_{t=0}=v_{i}^{(0)}(x),\left.v_{i}\right|_{x_{1}=0}=V_{i}\left(t, x^{\prime}\right), \quad(i=2, \ldots, r), \\
& \left.v_{i}\right|_{t=0}=V_{i}(x),\left.\frac{\partial}{\partial t} v_{i}\right|_{t=0}=v_{i}^{(1)}(x), \quad(i=r+1, \ldots, N),
\end{aligned}
$$

where $v_{i}^{(0)}(x),(i=1, \ldots, r)$ and $v_{i}^{(1)}(x),(i=r+1, \ldots, N)$ are defined in a neighborhood of the origin by

$$
\begin{gathered}
c\left(0,0, x^{\prime}\right) v_{1}^{(0)}(x)=x_{1} \widetilde{c}(0, x) v_{1}^{(0)}+\sum_{j=2}^{r} b_{1 j}(0, x ; 0,0,0) v_{j}^{(0)}(x) \\
+\sum_{j=r+1}^{N} b_{1 j}\left(0, x ; 0, \frac{\partial}{\partial x_{1}}, \frac{\partial}{\partial x^{\prime}}\right) V_{j}(x)+g_{1}(0, x)
\end{gathered}
$$




$$
\begin{array}{r}
\frac{\partial}{\partial x_{1}} v_{i}^{(0)}(x)=\sum_{j=1}^{N} b_{i j}\left(0, x ; 0,0, \frac{\partial}{\partial x^{\prime}}\right) v_{j}^{(0)}(x)+g_{i}(0, x), \\
\left.v_{i}^{(0)}(x)\right|_{x_{1}=0}=V_{i}\left(0, x^{\prime}\right), \\
v_{i}^{(1)}(x)=\sum_{j=1}^{N} b_{i j}\left(0, x ; 0, \frac{\partial}{\partial x_{1}}, \frac{\partial}{\partial x^{\prime}}\right) v_{j}^{(0)}(x)+g_{i}(0, x), \\
(i=r+1, \ldots, N),
\end{array}
$$

where $v_{i}^{(0)}(x)=V_{i}(x),(i=r+1, \ldots, N)$.

We note that $v_{i}^{(0)}(x),(i=1, \ldots, r)$ are defined uniquely by (6.13) in a neighborhood of the origin. (Cauchy-Kowalewski Theorem).

Clearly (6.11)-(6.12) has the same formal solution with that of (6.9) $-(4.6)$ in view of the construction of the problem (6.11)-(6.12).

Without loss of generality we may assume that the Goursat-data (6.12) are all zero.

Let

$$
\frac{M}{\left(1-\frac{t+x_{1}}{\rho}\right)\left(1-\frac{x_{2}+\cdots+x_{n}}{\rho}\right)}
$$

be a common majorant of the coefficients of differential appeared in the right hand side of (6.11). And the assumption, $p+c\left(0,0, x^{\prime}\right) \neq 0$ for any $p \in\{0,1,2, \ldots\}$, asserts the existence of a majorant $A\left(x^{\prime}\right)$ such that

$$
\frac{p+1}{p+\left(1+c\left(0,0, x^{\prime}\right)\right)} \ll A\left(x^{\prime}\right)
$$

for any $p \in\{0,1,2, \ldots\}$ in a neighborhood of the origin. Thus we have à majorant system of (6.11)

$$
\begin{aligned}
& \left\{t \frac{\partial^{2}}{\partial t^{2}}+\frac{\partial}{\partial t}\right\} w_{1}=\frac{N M A\left(x^{\prime}\right)}{\left(1-\frac{t+x_{1}}{\rho}\right)\left(1-\frac{x_{2}+\cdots+x_{n}}{\rho}\right)}\left[\left\{\sum_{k=1}^{n} t \frac{\partial^{2}}{\partial x_{k} \partial t}\right.\right. \\
& \left.+t \frac{\partial}{\partial t}+x_{1} \frac{\partial}{\partial t}+\sum_{l+|\nu| \leqq 2}\left(\frac{\partial}{\partial x_{1}}\right)^{l}\left(\frac{\partial}{\partial x^{\prime}}\right)^{\nu}\right\} w_{1}+\sum_{j=2}^{3}\left\{t \frac{\partial^{2}}{\partial t^{2}}\right. \\
& \left.\left.\quad+\sum_{k=1}^{n} t \frac{\partial^{2}}{\partial x_{k} \partial t}+\frac{\partial}{\partial t}+\sum_{l+|\nu| \leqq 2}\left(\frac{\partial}{\partial x_{1}}\right)^{l}\left(\frac{\partial}{\partial x^{\prime}}\right)^{\nu}\right\} w_{j}+c\right]
\end{aligned}
$$




$$
\begin{gathered}
\frac{\partial^{2}}{\partial x_{1} \partial t} w_{2}=\frac{N M}{\left(1-\frac{t+x_{1}}{\rho}\right)\left(1-\frac{x_{2}+\cdots+x_{n}}{\rho}\right)}\left[\sum _ { j = 1 } ^ { 3 } \left\{t \frac{\partial^{2}}{\partial t^{2}}+t \frac{\partial^{2}}{\partial x_{1} \partial t}\right.\right. \\
\left.\left.+\sum_{k=2}^{n} \frac{\partial^{2}}{\partial x_{k} \partial t}+\frac{\partial}{\partial t}+\sum_{l+|\nu| \leqq 2}\left(\frac{\partial}{\partial x_{1}}\right)^{l}\left(\frac{\partial}{\partial x^{\prime}}\right)^{\nu}\right\} w_{j}+c\right] \\
\frac{\partial^{2}}{\partial t^{2}} w_{3}=\frac{N M}{\left(1-\frac{t+x_{1}}{\rho}\right)\left(1-\frac{x_{2}+\cdots+x_{n}}{\rho}\right)}\left[\sum _ { j = 1 } ^ { 3 } \left\{t \frac{\partial^{2}}{\partial t^{2}}\right.\right. \\
\left.\left.+\sum_{\substack{i \leqq 1 \\
i+l|l| \leqq 2}}\left(\frac{\partial}{\partial t}\right)^{i}\left(\frac{\partial}{\partial x_{1}}\right)^{l}\left(\frac{\partial}{\partial x^{\prime}}\right)^{\nu}\right\} w_{j}+c\right], \\
\left.w_{1}\right|_{t=0}=\left.w_{2}\right|_{t=0}=\left.w_{2}\right|_{x_{1}=0}=\left.\left(\frac{\partial}{\partial t}\right)^{j} w_{3}\right|_{t=0}=0, \quad(j=0,1),
\end{gathered}
$$

where $c$ is a positive constant such that $c N M /\left(1-\frac{t+x_{1}}{\rho}\right)\left(1-\frac{x_{2}+\cdots+x_{n}}{\rho}\right)$ is a common majorant of $h_{1}$ and $\frac{\partial}{\partial t} g_{i}$, and we have $w_{i},(i=1,2,3)$ so that $w_{1}$ is a majorant of $v_{1}, w_{2}$ is a majorant of $v_{j},(j=2, \ldots, r)$ and $w_{3}$ is a majorant of $v_{j},(j=r+1, \ldots, N)$, respectively.

It is obvious that the formal solution of (6.15)-(6.16) is majorant of that of (6.11)-(6.12) in view of the construction of the formal solution.

Now we note that (6.15)-(6.16) is a problem considered in Lemma 6.1 (see Remark 6.1), then there exists a unique solution of (6.15)-(6.16) in a neighborhood of the origin. Thus we proved the lemma.

Q.E.D.

Proof of Theorem 4.2. We shall prove the theorem for the system (3.6) replacing (1.1)-(1.2). We prove the sufficiency, since the necessity is obvious. Let us consider the following Goursat-problem to (3.6):

$$
\begin{aligned}
& \left.v_{i}\right|_{x_{1}=0}=V_{i}\left(0, x^{\prime}\right)+t x_{i}\left(t, x^{\prime}\right), \quad(i=2, \ldots, r), \\
& \left.v_{i}\right|_{t=0}=V_{i}(x), \quad(i=r+1, \ldots, N),
\end{aligned}
$$

where $\vec{V}(x)={ }^{t}\left(V_{1}(x), \ldots, V_{N}(x)\right)$ is the initial-data of (3.6) and $x_{i}\left(t, x^{\prime}\right)$ are arbitrary analytic functions at $t$ and $x^{\prime}$. Then there exists a unique 
solution $v_{i}(t, x),(i=1, \ldots, N)$ in a neighborhood of the origin. We can prove that this solution is also a solution of (3.6) with the initial-data $\vec{V}(x)$. In fact, it suffices to show that $v_{i}^{(0)}(x) \equiv v_{i}(0, x)=V_{i}(x), \quad(i=1$, $\ldots, r)$. We note that $v_{i}(0, x),(i=1, \ldots, r)$ is a solution of the initialvalue problem (6.13).

On the other hand it is obvious that $V_{i}(x),(i=1, \ldots, r)$ is a solution of the initial-value problem (6.13), since (6.13) is nothing but the compatibility condition (4.7) if we substitute $v_{i}^{(0)}(x)$ by $V_{i}(x),(i=1, \ldots, r)$. Thus we have that $v_{i}(0, x)=V_{i}(x),(i=1, \ldots, r)$ in a neighborhood of the origin (Cauchy-Kowalewski Theorem).

The non-uniqueness of the solution is obvious in view of the arbitrariness of $x_{i}\left(t, x^{\prime}\right),(i=2, \ldots, r)$ in $(6.17)$.

Q.E.D.

\section{§7. Proof of Theorem 4.3}

Let

$$
v_{i}(t, x)=\sum_{p, q \geqq 0} v_{i}^{(p, q)}\left(x^{\prime}\right) \frac{t^{p}}{p !} \frac{x_{i}^{q}}{q !}, \quad(i=1, \ldots, N),
$$

be the formal solution of the problem (3.6)-(4.6).

At first, we prove the theorem in the case where $a(0, x) \equiv 0$, and $c(0,0)=0$ and $c(0, x) \neq 0$. Let us construct the formal solution (7.1), then we see that $v_{1}^{(0,0)}\left(x^{\prime}\right)$ should satisfy

$$
\begin{aligned}
& c\left(0,0, x^{\prime}\right) v_{1}^{(0,0)}\left(x^{\prime}\right)+\left.\frac{\partial}{\partial x_{1}} V_{r+1}(x)\right|_{x_{1}=0} \\
& =\sum_{j=2}^{r} b_{1 j}\left(0,0, x^{\prime} ; 0,0,0\right) v_{j}\left(0, x^{\prime}\right) \\
& \quad+\left.\sum_{j=r+1}^{N} b_{1 j}\left(0,0, x^{\prime} ; 0,0, \frac{\partial}{\partial x^{\prime}}\right) V_{j}(x)\right|_{x_{1}=0} \\
& +<\vec{l}_{12}(x), f(0, x)>\left.\right|_{x_{1}=0 .}
\end{aligned}
$$

Thus we see that the Goursat-data (4.6) should satisfy some relation at $x^{\prime}=0$, since $c(0,0,0)=0$. 
Now we prove the theorem in the case where $a(0, x) \equiv 0, c(0, x) \neq 0$. Without loss of generality we may rewrite the system (3.6) as follows,

$$
\begin{gathered}
v_{1}=\left\{t^{2} a(t, x) \frac{\partial}{\partial t}+\sum_{k=1}^{n} t b_{k}(t, x) \frac{\partial}{\partial x_{k}}\right\} v_{1}+d(x) \frac{\partial}{\partial x_{1}} v_{r+1} \\
+\sum_{j=2}^{r} b_{1 j}\left(t, x ; t \frac{\partial}{\partial t}, t \frac{\partial}{\partial x_{1}}, t \frac{\partial}{\partial x^{\prime}}\right) v_{j} \\
+\sum_{j=r+1}^{N} b_{1 j}\left(t, x ; t \frac{\partial}{\partial t}, t \frac{\partial}{\partial x_{1}}, \frac{\partial}{\partial x^{\prime}}\right) v_{j}+g_{1}(t, x),
\end{gathered}
$$

$$
\begin{aligned}
& \frac{\partial}{\partial x_{1}} v_{i}=\sum_{j=1}^{N} b_{i j}\left(t, x ; t \frac{\partial}{\partial t}, t \frac{\partial}{\partial x_{1}}, \frac{\partial}{\partial x^{\prime}}\right) v_{j}+g_{i}(t, x), \quad(i=2, \ldots, r), \\
& \frac{\partial}{\partial t} v_{r+1}=-\frac{\partial}{\partial x_{1}} v_{1}+\sum_{j=1}^{N} b_{r+1, j}\left(t, x ; t \frac{\partial}{\partial t}, t \frac{\partial}{\partial x_{1}}, \frac{\partial}{\partial x^{\prime}}\right) v_{j}+g_{r+1}, \\
& \frac{\partial}{\partial t} v_{i}=\sum_{j=1}^{N} b_{i j}\left(t, x ; t \frac{\partial}{\partial t}, \frac{\partial}{\partial x_{1}}, \frac{\partial}{\partial x^{\prime}}\right) v_{j}+g_{i}, \quad(i=r+2, \ldots, N),
\end{aligned}
$$

where $d(0) \neq 0$.

We give only a sketch of the proof, since an exact discription is more complicated.

Our purpose is to construct a Goursat-data (4.6) so that the formal solution (7.1) is not analytic in any neighborhood of the origin. We note that the formal solution (7.1) can be constructed uniquely according to the assumption.

Now let us consider the following Goursat-data,

$$
\begin{aligned}
& \left.v_{i}\right|_{x_{1}=0}=0, \quad(i=2, \ldots, r), \\
& \left.v_{r+1}\right|_{t=0}=\sum_{q \geqq 0} v_{r+1}^{(0,2 q+1)} \frac{x_{1}^{2 q+1}}{(2 q+1) !} \\
& \left.v_{i}\right|_{t=0}=0, \quad(i=r+2, \ldots, N)
\end{aligned}
$$

where $v_{r+1}^{(0,2 q+1)}$ are defined later so that the formal solution (7.1) is not analytic in any neighborhood of the origin.

Let 


$$
v_{i}(t, x)=\sum_{p, q \geqq 0} v_{i}^{(p, q)}\left(x^{\prime}\right) \frac{t^{p}}{p !} \frac{x_{1}^{q}}{q !}, \quad(i=1, \ldots, N),
$$

be the formal solution of the problem (7.2)-(7.3), and let

$$
g_{i}(t, x)=\sum_{p, q \geqq 0} g_{i}^{(p, q)}\left(x^{\prime}\right) \frac{t^{p}}{p !} \frac{x_{1}^{q}}{q !}, \quad(i=1, \ldots, N) .
$$

Then we have easily

$$
\begin{aligned}
v_{1}^{(p, 0)}\left(x^{\prime}\right)=( & \left.-d\left(0, x^{\prime}\right)\right)^{p} d\left(0, x^{\prime}\right) v_{r+1}^{(0,2 p+1)}+l_{p}\left(v_{r+1}^{(0, q)} ; q<2 p\right) \\
& +\mathscr{L}_{p}\left(x^{\prime} ; \frac{\partial}{\partial x^{\prime}}\right)\left(g_{i}^{\left(p^{\prime}, q^{\prime}\right)}\left(x^{\prime}\right)\right),
\end{aligned}
$$

where $l_{p}$ denotes a linear combination of $v_{r^{+1}}^{(0, q)},(q<2 p)$, and $\mathscr{L}_{p}\left(x^{\prime} ; \frac{\partial}{\partial x^{\prime}}\right)$ denotes a suitable differential operator.

Now we define $v_{r+1}^{(0,2 p+1)}$ by

$$
v_{r+1}^{(0,2 p+1)}=-\eta^{p+1} e^{i \theta} p(2 p+1) !
$$

where $\eta=-d(0,0)^{-1}$, and the argument $\theta_{p}$ is defined by

$$
\theta_{p}=\arg \cdot\left(\left.l_{p}\left(v_{r+1}^{(0, q)} ; q<2 p\right)\right|_{x^{\prime}=0}+\left.\mathscr{L}_{p}\left(0, \frac{\partial}{\partial x^{\prime}}\right)\left(g_{i}^{\left(p^{\prime}, q^{\prime}\right)}\right)\right|_{x^{\prime}=0}\right)
$$

Then we have

$$
\left|v_{1}^{(p, 0)}(0)\right| \geqq(2 p+1) !
$$

This implies

$$
\sum_{p \geqq 0}\left|v_{1}^{(p, 0)}(0)\right| \frac{t^{p}}{p !} \geqq \sum_{p \geqq 0} \frac{(2 p+1) !}{p !} t^{p}
$$

This completes the proof.

Q.E.D.

\section{§8. Statements of Theorems in the Case Where $r=1$}

In this section we give only the results, since the proofs are similar to those in the previous sections and in $[6]$. 
Theorem 8.1. We assume (1.4) and

$$
a(0, x) \equiv 0, \quad c(0, x) \equiv 0 .
$$

Then for the existence of a solution of the problem (1.1)-(1.2), the following conditions are necessary and sufficient; the first compatibility condition

$$
<\vec{l}_{12}(x), \mathscr{L}\left(0, x ; \frac{\partial}{\partial x}\right) \vec{U}(x)-\vec{f}(0, x)>=0
$$

and the second compatibility condition

$$
\begin{aligned}
<\vec{l}_{12}(x),\left(A^{(1)}(x)\right. & \left.+\mathscr{L}\left(0, x ; \frac{\partial}{\partial x}\right)\right) \vec{u}^{(1)}(x) \\
& +\mathscr{L}^{(1)}\left(x ; \frac{\partial}{\partial x}\right) \vec{U}(x)-\vec{f}^{(1)}(x)>=0,
\end{aligned}
$$

where $\vec{u}^{(1)}(x)$ is a special solution of the equation

$$
A(0, x) \vec{u}^{(1)}(x)+\mathscr{L}\left(0, x ; \frac{\partial}{\partial x}\right) \vec{U}(x)=\vec{f}(0, x)
$$

In the above theorem the necessity follows immediately from the construction of the formal solution of type

$$
\vec{u}(t, x)=\sum_{p \geqq 0} \vec{u}^{(p)}(x) t^{p} / p ! 。
$$

Let us recall that we are assuming (1.4). Contrary to (8.1), we now assume

$$
a(0, x) \equiv 0, \quad c(0, x) \neq 0 .
$$

If the initial-value problem (1.1)-(1.2) admits an analytic solution $\vec{u}(t, x)$, then the initial-data (1.2) should satisfy the following condition:

$$
<\vec{l}_{12}(x), \mathscr{L}\left(0, x ; \frac{\partial}{\partial x}\right) \vec{U}(x)-\vec{f}(0, x)>=0
$$

Theorem 8.2. Under the assumptions (1.4) and (8.5), (8.6) is not 
always sufficient for the existence of solutions. Precisely we can find an initial-data satisfying (8.6) such that there can not exist analytic solution in any neighborhood of the origin.

Theorem 8.3. Under the assumptions (1.4) and

$$
a(0, x) \neq 0, \quad p a(0, x)+c(0, x) \neq 0,
$$

for any $p \in\{0,1,2, \ldots\},(8.6)$ is necessary and also sufficient in order that there exists a solution of the problem (1.1)-(1.2). In this case the solution is unique.

Theorem 8.4. We now assume (1.4) and

$$
a(0, x) \neq 0, \quad p_{0} a(0, x)+c(0, x) \equiv 0,
$$

for some $p_{0} \in\{0,1,2, \ldots\}$. If the case where $p_{0}=0,(8.6)$ is necessary and also sufficient in order that there exists a solution of the problem (1.1)(1.2) in a neighborhood of the origin. In this case the solution is unique.

If the case where $p_{0} \geqq 1,(8.6)$ and the following compatibility conditions are necessary and sufficient in order to exist at least one solution of the problem (1.1)-(1.2) in a neighborhood of the origin. In this case the solution is not unique;

$$
\begin{gathered}
\left(\frac{\partial}{\partial t}\right)^{p_{0}}<\vec{l}_{12}(x),\left\{A(t, x) \frac{\partial}{\partial t}+\mathscr{L}\left(t, x ; \frac{\partial}{\partial x}\right)\right\} \overrightarrow{\vec{u}}(t, x) \\
-\vec{f}(t, x)>\left.\right|_{t=0}=0,
\end{gathered}
$$

where $\overrightarrow{\vec{u}}(t, x)=\sum_{p=0}^{p_{0}} \vec{u}^{(p)}(x) t^{p} / p$ ! is defined followingly; $\vec{u}^{(0)}(x)=\vec{U}(x), \vec{u}^{(p)}(x)$, $\left(1 \leqq p \leqq p_{0}-1\right)$ are uniquely detemined step by step by the construction of the formal solution of type (8.4) and $\vec{u}^{\left(p_{0}\right)}(x)$ is a special solution of the equation

$$
A(0, x) \frac{\vec{u}^{\left(p_{0}\right)}}{\left(p_{0}-1\right) !}+\left.\left(\frac{\partial}{\partial t}\right)^{p_{0}-1}\left\{A(t, x) \overrightarrow{\tilde{\tilde{u}}}(t, x)+\mathscr{L}\left(t, x ; \frac{\partial}{\partial x}\right) \overrightarrow{\tilde{\tilde{u}}}\right\}\right|_{t=0}=0
$$

wehre $\overrightarrow{\tilde{\tilde{u}}}(t, x)=\sum_{p=0}^{p_{0}-1} \vec{u}^{(p)}(x) t^{p} / p !$ 
Remark 8.1. In Theorem 8.3 and Theorem 8.4, we need not assume that the initial surface $t=0$ is double characteristic. More precisely it suffices to assume the following;

$$
\operatorname{rank} A(0, x)=N-1, \quad \sum_{k=1}^{n}\left|\frac{\partial H}{\partial \xi_{k}}(0, x ; 1,0)\right|=0 .
$$

\section{Appendix}

In the previous sections we assumed (3. Ass.), we study in this section the case where we do not assume (3. Ass.) but the following assumption
(A. Ass.)
$<\vec{l}_{i 2}(x), B_{k}(0, x) \vec{r}(x)>=0$
$(i=1, \ldots, r ; k=1, \ldots, n)$

Then we have

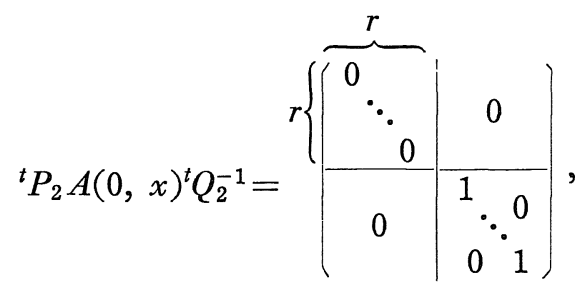

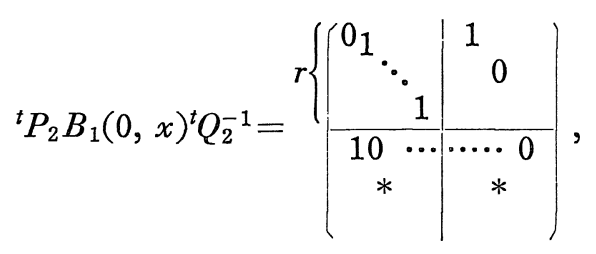

$$
\begin{aligned}
& { }^{t} P_{2} B_{k}(0, x)^{t} Q_{2}^{-1}=r\left(\begin{array}{ll|c}
0 & & \\
\vdots & * & * \\
0 & & \\
\hline & * & *
\end{array}\right), \quad(k=2, \ldots, n) .
\end{aligned}
$$

(See the notation in $\S 3$. )

Remark A.1. In the case where $r=2$, we easily see that the assump- 
tion (1.4)' implies (3. Ass.) or (A. Ass.).

Now we transform the unknown function $\vec{u}$ to $\vec{v}$ by $\vec{v}={ }^{t} Q_{2} \vec{u}$, then we have the following system;

$$
\begin{gathered}
\left\{t a_{1}(t, x) \frac{\partial}{\partial t}+\sum_{k=1}^{n} t b_{1 k}(t, x) \frac{\partial}{\partial x_{k}}+c_{1}(t, x)\right\} v_{1}+\frac{\partial}{\partial x_{1}} v_{r+1} \\
=\sum_{j=2}^{N} b_{1 j}\left(t, x ; t \frac{\partial}{\partial t}, t \frac{\partial}{\partial x_{1}}, \frac{\partial}{\partial x^{\prime}}\right) v_{j}+<\vec{l}_{12}, \vec{f}>, \\
\frac{\partial}{\partial x_{1}} v_{i}=\left\{t a_{i}(t, x) \frac{\partial}{\partial t}+\sum_{k=1}^{n} t b_{i k}(t, x) \frac{\partial}{\partial x_{k}}+c_{i}(t, x)\right\} v_{1} \\
+\sum_{j=2}^{N} b_{i j}\left(t, x ; t \frac{\partial}{\partial t}, t \frac{\partial}{\partial x_{1}}, \frac{\partial}{\partial x^{\prime}}\right) v_{j}+<\vec{l}_{i 2}, \vec{f}>, \\
\begin{array}{c}
\frac{\partial}{\partial t} v_{r+1}+\frac{\partial}{\partial x_{1}} v_{1}=\sum_{j=1}^{N} b_{p+1} j\left(t, x ; t \frac{\partial}{\partial t}, t \frac{\partial}{\partial x_{1}}, \frac{\partial}{\partial x^{\prime}}\right) v_{j} \\
\left.\quad+<\vec{a}_{r+1,2}, \vec{f}>, r\right), \\
\frac{\partial}{\partial t} v_{i}=\sum_{j=1}^{N} b_{i j}\left(t, x ; t \frac{\partial}{\partial t}, \frac{\partial}{\partial x_{1}}, \frac{\partial}{\partial x^{\prime}}\right) v_{j}+<\vec{a}_{i 2}, \vec{f}>, \\
(i=r+2, \ldots, N) .
\end{array}
\end{gathered}
$$

Let us show that we can not obtain a corresponding result to Theorem 4.1 by a simple example.

Example. Let us consider the following system,

$$
\frac{\partial}{\partial x_{1}} u_{3}=a \frac{\partial}{\partial x_{2}} u_{2}+b \frac{\partial}{\partial x_{2}} u_{3}+\mathscr{L}_{1}\left(u_{2}, u_{3}\right)
$$

(E)

$$
\begin{aligned}
& \frac{\partial}{\partial x_{1}} u_{2}=c \frac{\partial}{\partial x_{2}} u_{2}+d \frac{\partial}{\partial x_{2}} u_{3}+\mathscr{L}_{2}\left(u_{2}, u_{3}\right), \\
& \frac{\partial}{\partial t} u_{3}=\frac{\partial}{\partial x_{1}} u_{1}+e \frac{\partial}{\partial x_{2}} u_{1}+f \frac{\partial}{\partial x_{2}} u_{2}+g \frac{\partial}{\partial x_{2}} u_{3}+\mathscr{L}_{3}\left(u_{1}, u_{2}, u_{3}\right),
\end{aligned}
$$


where $a(\neq 0), b, \ldots, f$ and $g$ are complex numbers and $\mathscr{L}_{i},(i=1,2,3)$ denote linear combinations.

Now let us assume that there exists a solution of $(\mathrm{E}), u_{i}(t, x)=$ $\sum_{p \geqq 0} u_{i}^{(p)}(x) t^{p} / p !,(i=1,2,3)$. Then we easily see that we can not obtain relations between $u_{i}^{(0)}(x),(i=1,2,3)$. On the other hand, we know that we can not give a datum of $u_{1}$ at $t=0$.

We now assume

$$
<\vec{l}_{i 2}(x), \mathscr{L}\left(0, x ; \frac{\partial}{\partial x}\right) \vec{r}(x)>=0, \quad(i=1, \ldots, r)
$$

which implies $c_{i}(0, x)=0,(i=1, \ldots, r)$ in the system (A.2).

Under the assumption (A.3), we shall consider the following initialvalue problem to (1.1);

$$
<\vec{b}_{i 2}(x), \vec{u}>\left.\right|_{t=0}=V_{i}(x), \quad(i=2, \cdots, N) .
$$

Theorem A.1. Under the assumptions (A. Ass.) and (A.3), the following conditions are necessary and sufficient in order to exist at least one solution of the problem (1.1)-(A.4) in a neighborhood of the origin;

$$
<\vec{l}_{i 2}(x), \mathscr{L}\left(0, x ; \frac{\partial}{\partial x}\right) \vec{u}(t, x)-\vec{f}(t, x)>\left.\right|_{t=0}=0, \quad(i=1, \ldots, r) .
$$

Theorem A.1 follows immediately from the following lemma.

Lemma A.1. (Goursat-problem) Let us consider the following problem to the system (A.2), then there exists a unique solution in a neighborhood of the origin;

$$
\begin{array}{ll}
\left.v_{i}\right|_{x_{1}=0}=V_{i}\left(t, x^{\prime}\right), & (i=1, \cdots, r+1), \\
\left.v_{i}\right|_{t=0}=V_{i}(x), & (i=r+2, \ldots, N) .
\end{array}
$$

We can prove the lemma as the same way as Lemma 4.2 , or see L. Gårding ( $[2]$, Théorème 3.1).

We now assume

$$
a_{1}(0, x) \neq 0, \quad p a_{1}(0, x)+c_{1}(0, x) \neq 0 \quad \text { for } p \in\{0,1,2, \ldots\},
$$


then we have a corresponding result to Theorem 4.2 .

Theorem A.2. Under the assumptions (A. Ass.) and (A.7), the following conditions are necessary and sufficient for the existence of a solution of the problem (1.1)-(1.2) in a neighborhood of the origin;

$$
<\vec{l}_{i 2}(x), \mathscr{L}\left(0, x ; \frac{\partial}{\partial x}\right) \vec{U}(x)-\vec{f}(0, x)>=0, \quad(i=1, \cdots, r)
$$

In order to prove the theorem, it suffices to prove the following lemma (see $\S 6$ ).

Lemma A.2. Let us consider the following Goursat-problem, then there exists a unique solution in a neighborhood of the origin;

$$
\begin{gathered}
\left(t \frac{\partial}{\partial t}+1\right) v_{1}=M_{1}\left(x^{\prime}\right)\left[\left\{t\left(t+x_{1}\right) \frac{\partial}{\partial t}+\sum_{k=1}^{n} t \frac{\partial}{\partial x_{k}}+t+x_{1}\right\} v_{1}\right. \\
\left.+t \frac{\partial}{\partial x_{1}} v_{2}+\frac{\partial}{\partial x_{1}} v_{3}+\sum_{j=2}^{3}\left(t \frac{\partial}{\partial t}+\sum_{k=2}^{n} \frac{\partial}{\partial x_{k}}+1\right) v_{j}\right]+F_{1}\left(x^{\prime}\right)
\end{gathered}
$$

$$
\begin{aligned}
& \frac{\partial}{\partial t} v_{2}=M_{2}\left(x^{\prime}\right)\left[x_{1} \frac{\partial}{\partial x_{1}} v_{2}+\left(t \frac{\partial}{\partial t}+\sum_{k=1}^{n} t \frac{\partial}{\partial x_{k}}+1\right) v_{1}\right. \\
&\left.+\sum_{j=2}^{3}\left(t \frac{\partial}{\partial t}+t \frac{\partial}{\partial x_{1}}+\sum_{k=2}^{n} \frac{\partial}{\partial x_{k}}+1\right) v_{j}\right]+F_{2}\left(x^{\prime}\right) \\
& \frac{\partial}{\partial t} v_{3}=M_{3}\left(x^{\prime}\right)\left[x_{1} \frac{\partial}{\partial t} v_{3}+\sum_{j=1}^{3}\left(t \frac{\partial}{\partial t}+\sum_{k=1}^{n} \frac{\partial}{\partial x_{k}}+1\right) v_{j}\right]+F_{3}\left(x^{\prime}\right) .
\end{aligned}
$$

$$
\left.v_{2}\right|_{x_{1}=0}=\left.v_{3}\right|_{t=0}=0
$$

Proof of Lemma A.2. Let $v_{i}=\sum_{p, q \geqq 0} v_{i}^{(p, q)}\left(x^{\prime}\right) t^{p} x_{1}^{q} / p ! q !,(i=1,2,3)$ be the formal solution of (A.9)-(A.10). Since $M_{i}\left(x^{\prime}\right)$ and $F_{i}\left(x^{\prime}\right),(i=1,2,3)$ are analytic functions at $x^{\prime}$, we now assume

$$
\begin{aligned}
& \left|\left(\frac{\partial}{\partial x^{\prime}}\right)^{\alpha} M_{i}\left(x^{\prime}\right)\right|,\left|\left(\frac{\partial}{\partial x^{\prime}}\right)^{\alpha}\left\{M_{i}\left(x^{\prime}\right) M_{j}\left(x^{\prime}\right)\right\}\right| \leqq \frac{|\alpha| !}{(3 \rho)^{|\alpha|}} M, \\
& \left|\left(\frac{\partial}{\partial x^{\prime}}\right)^{\alpha} F_{i}\left(x^{\prime}\right)\right| \leqq \frac{|\alpha| !}{\rho^{|\alpha|}} A, \quad(i, j=1,2,3)
\end{aligned}
$$


for some positive constants $M$ and $A$ in $\left|x^{\prime}\right|<\delta(\delta>0)$.

Then we can prove the following estimates by induction on $p$ and $q$,

$$
\left|\left(\frac{\partial}{\partial x^{\prime}}\right)^{\alpha} v_{1}^{(p, q)}\left(x^{\prime}\right)\right| \leqq \frac{(2 p+q+1+|\alpha|) !}{(p+1) !} C^{7 p+3 q+2+|\alpha|} A
$$

$$
\left|\left(\frac{\partial}{\partial x^{\prime}}\right)^{\alpha} v_{i}^{(p, q)}\left(x^{\prime}\right)\right| \leqq \frac{(2 p+q+|\alpha|) !}{p !} C^{7 p+3 q+|\alpha|} A, \quad(i=2,3),
$$

for some positive constant $C$ in $\left|x^{\prime}\right|<\delta$.

We must pay attention when we estimate $\left(\frac{\partial}{\partial x^{\prime}}\right)^{\alpha} v_{1}^{(p, q)}$, because of the fact that $\frac{\partial}{\partial x_{1}} v_{3}$ appear in the right hand side of the first equation in the system (A.9). This completes the proof.

\section{References}

[1] Duff, G.F.D., Mixed problems for linear systems of first order equations, Canadian J. Math. 10 (1958), 127-160.

[2] Gårding, L., Une variante de la méthode de majoration de Cauchy, Acta Math. 114 (1965), 143-158.

[3] Goursat, E., Leçon sur l'intégration des équations aux derivées partielles du seconds ordre à deux variables indépendents, Hermann, Paris, I (1896), II (1898).

[4] Hadamard, J., La Propagation des Ondes, Paris, 1903.

[5] - Le Problème de Cauchy, Paris, 1932.

[6] Hasegawa, Y., On the initial-value problems with data on a double characteristic, J. Math. Kyoto Univ. 11 (1971), 357-372.

[7] Hörmander, L., Linear Partial Differential Operators, Springer-Verlag, 1963.

[8] Mizohata, S., Solutions nulles et solutions non analytiques, J. Math. Kyoto Univ. 1 (1962), 271-302.

[9] Vaillant, J., Données de Cauchy portées par une caractéristique double, ..., J. Math. Pures Appl. 47 (1968), 1-40.

[10] Problème de Cauchy caractéristique et .., C. R. Acad. Sci. Paris, 267 (1968), 891-894.

[11] Matsuura, S., On general systems of partial differential operators with constant coefficients, J. Math. Soc. Japan, 13 (1961), 94-103.

[12] Petrowsky, I. G., Sur l'analyticité des solutions des systèmes d'équations différentielles, Mat. Sb. 5 (47) (1939), 3-70. 